\title{
EXACT CATEGORIES AND DUALITY
}

\author{
BY \\ D. A. BUCHSBAUM
}

\section{INTRODUCTION}

Throughout the book Homological algebra, by H. Cartan and S. Eilenberg, the authors dealt with functors defined on categories of modules over certain rings and whose values again were modules over a ring. It will be shown in this paper that the theory may be generalized to functors defined on abstract categories, and whose values are again in such abstract categories. An abstract treatment such as this has several advantages. We list a few:

(1) The dualities of the type

$$
\begin{aligned}
& \text { Kernel }- \text { cokernel } \\
& \text { Projective }- \text { injective } \\
& Z(A)-Z^{\prime}(A),
\end{aligned}
$$

originally suggested by MacLane [4], may now be formulated as explicit mathematical theorems.

(2) In treating derived functors, it suffices to consider left derived functors of a covariant functor of several variables; all other types needed may then be obtained by a dualization process.

(3) Further applications of the theory of derived functors are bound to show that the consideration of modules over a ring $\Lambda$ will be insufficient. Rings with additional structure such as grading, differentiation, topology, etc., will have to be considered. With the theory developed abstractly, these generalizations are readily available.

The paper is divided into four parts. Part I deals with basic definitions, duality, and fundamental lemmas. We make no attempt to prove or even state many of the necessary trivia which are used throughout.

Part II is rather short, due to the facts that most of the results follow trivially from Part I, and the definitions are identical with those in [1]. Connected sequences of functors were included in $\$ 4$ since they are so similar to homology sequences, and the proof of 4.1 was given to show that it is independent of Axiom V, which is not apparent from [1].

Part III is devoted to the abstract treatment of the fundamental concepts in [1]. Theorem 5.1, however, is proved in its full generality so as to be applicable in the theory of sheaves.

Part IV contains three applications of a purely algebraic nature. We desist from giving applications to theory of sheaves as these would be fragmentary.

Received by the editors November 13, 1954. 
The following treatment has some points in common with that of MacLane [4].

\section{Part I. Exact Categories}

1. Definition of exact categories. An exact category $A$ is given by the following four data:

(i) a collection of objects $A$,

(ii) a distinguished object $\varnothing$, called the zero object,

(iii) an abelian group $H(A, B)$ given for any pair of objects $A, B$ in $\mathcal{A}$. The elements $\phi \in H(A, B)$ will be called maps. We shall frequently write $\phi: A \rightarrow B$ instead of $\phi \in H(A, B)$. The zero element of any of the groups $H(A, B)$ will be denoted by 0 ;

(iv) a homomorphism $H(B, C) \otimes H(A, B) \rightarrow H(A, C)$ given for each triple of objects $A, B, C \in \mathcal{A}$. The image of $\psi \otimes \phi$ in $H(A, C)$ will be denoted by $\psi \phi$ and will be called the composition of $\psi$ and $\phi$.

The primitive terms of (i)-(iv) are subjected to four axioms.

Axiom I. If $\alpha: A \rightarrow B, \beta: B \rightarrow C, \gamma: C \rightarrow D$, then $\gamma(\beta \alpha)=(\gamma \beta) \alpha$.

Axiom II. $H(\varnothing, \varnothing)=0$.

Axiom III. For each $A \in A$ there is a map $e_{A}: A \rightarrow A$ such that $e_{A} \beta=\beta$ for each $\beta: B \rightarrow A$ and $\gamma e_{A}=\gamma$ for each $\gamma: A \rightarrow C$.

It is easy to verify that $H(A, \varnothing)=0=H(\varnothing, A)$ for all $A \in \mathcal{A}$ and that the identity map $e_{A}$ of Axiom III is unique.

A map $\phi: A \rightarrow B$ will be called an equivalence if there exists a map $\phi^{\prime}: B \rightarrow A$ such that $\phi^{\prime} \phi=e_{A}, \phi \phi^{\prime}=e_{B}$. It is easy to see that $\phi^{\prime}$ is unique; we write $\phi^{\prime}=\phi^{-1}$. Clearly $\phi^{-1}$ also is an equivalence and $\left(\phi^{-1}\right)^{-1}=\phi$. If $\psi: B \rightarrow C$ is another equivalence, then $\psi \phi$ also is an equivalence and $(\psi \phi)^{-1}=\phi^{-1} \psi^{-1}$.

Definition. We shall say that the pair of maps

$$
A \stackrel{\alpha}{\rightarrow} B \stackrel{\beta}{\rightarrow} C
$$

has property $(\mathrm{E})$ if the following conditions hold

(1) $\beta \alpha=0$.

(2) If $\alpha^{\prime}: A^{\prime} \rightarrow B$ and $\beta \alpha^{\prime}=0$, then there exists a unique $\gamma: A^{\prime} \rightarrow A$ with $\alpha^{\prime}=\alpha \gamma$.

(3) If $\beta^{\prime}: B \rightarrow C^{\prime}$ and $\beta^{\prime} \alpha=0$, then there exists a unique $\delta: C \rightarrow C^{\prime}$ with $\beta^{\prime}=\delta \beta$.

We are now ready to state

Axiom IV. For any map $\alpha: A \rightarrow B$ there exist objects $K, I, I^{\prime}, F$ and maps such that

$$
K \stackrel{\sigma}{\rightarrow} A \stackrel{\tau}{\rightarrow} I \stackrel{\theta}{\rightarrow} I^{\prime} \stackrel{\kappa}{\rightarrow} B \stackrel{\pi}{\rightarrow} F
$$

such that

(4) $\alpha=\kappa \theta \tau$, 
(5) $\theta$ is an equivalence,

(6) $K \rightarrow^{\sigma} A \rightarrow^{\top} I$ has property (E),

(7) $I^{\prime} \rightarrow{ }^{\star} B \rightarrow{ }^{\top} F$ has property (E).

Lemma 1.1. Let $A \rightarrow^{\alpha} B \rightarrow^{\beta} C$ have property (E), and let $D \rightarrow^{\sigma} A\left(C \rightarrow^{\top} F\right)$ be such that $\alpha \sigma=0(\tau \beta=0)$. Then $\sigma=0(\tau=0)$.

THEOREM 1.2. If $K_{1} \rightarrow^{\sigma_{1}} A \rightarrow_{:}^{r_{1}} I_{1} \rightarrow{ }^{\theta_{1}} I_{1}^{\prime} \rightarrow^{\alpha_{1}} B \rightarrow^{\pi_{1}} F_{1}$ also satisfy (4)-(7), then there exist unique maps $\xi, \eta, \zeta, \omega$ such that the diagram

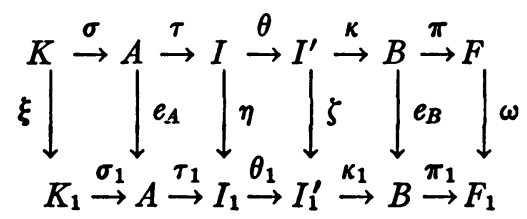

is commutative. The maps $\xi, \eta, \zeta, \omega$ are equivalences.

Proof. Since $\kappa_{1} \theta_{1} \tau_{1} \sigma=\alpha \sigma=\kappa \theta \tau \sigma=0$ implies that $\tau_{1} \sigma=0$, we know there is a unique $\xi: K \rightarrow K_{1}$ such that $\sigma=\sigma_{1} \xi$. Also we know there is a unique $\eta: I \rightarrow I_{1}$ such that $\tau_{1}=\eta \tau$. Similarly we obtain $\zeta$ and $\omega$ with $\kappa=\kappa_{1} \zeta$ and $\pi_{1}=\omega \pi$. Now we must show that $\theta_{1} \eta=\zeta \theta$. But

$$
\kappa_{1} \theta_{1} \eta \tau=\kappa_{1} \theta_{1} \tau_{1}=\alpha=\kappa \theta \tau=\kappa_{1} \zeta \theta \tau .
$$

Thus $\kappa_{1}\left(\theta_{1} \eta-\zeta \theta\right) \tau=0$ which, by Lemma 1.1, implies that $\theta_{1} \eta=\zeta \theta$.

It is easy to show that $\xi, \eta, \zeta, \omega$ are equivalences.

2. Exact sequences. In view of Theorem 1.2, we shall call the pairs $(K, \sigma),(I, \tau),\left(I^{\prime}, \kappa\right)$, and $(F, \pi)$ the kernel, coimage, image and cokernel of $\alpha$ respectively. The sense in which these notions are uniquely associated with $\alpha$ is clear from Theorem 1.2.

Definition. A sequence

$$
A_{m} \stackrel{\alpha_{m}}{\rightarrow} A_{m+1} \rightarrow \cdots \stackrel{\alpha_{n-1}}{\longrightarrow} A_{n}
$$$$
m+1<n
$$

is exact if $\operatorname{Ker} \alpha_{q}=\operatorname{Im} \alpha_{q-1} m<q<n$.

We now obtain the crucial

ThEOREM 2.1. A necessary and sufficient condition that

$$
A \stackrel{\alpha}{\rightarrow} B \stackrel{\beta}{\rightarrow} C
$$

have property $(\mathrm{E})$ is that

$$
\varnothing \rightarrow A \stackrel{\alpha}{\rightarrow} B \stackrel{\beta}{\rightarrow} C \rightarrow \varnothing
$$

be exact.

Proof. Suppose $A \rightarrow^{\alpha} B \rightarrow{ }^{\beta} C$ has property (E). Then we must show that 
$\varnothing \rightarrow A \rightarrow^{\alpha} B, A \rightarrow^{\alpha} B \rightarrow^{\beta} C, B \rightarrow^{\beta} C \rightarrow \varnothing$ are all exact. Let us first consider $A \rightarrow{ }^{\alpha} B \rightarrow^{\beta} C$, and show that $\operatorname{Ker} \beta=\operatorname{Im} \alpha$. The decompositions of $\alpha$ and $\beta$ can be taken to be

$$
\begin{aligned}
& \varnothing \rightarrow A \stackrel{e_{A}}{\rightarrow} A \stackrel{e_{A}}{\rightarrow} A \stackrel{\alpha}{\rightarrow} B \stackrel{\beta}{\rightarrow} C, \\
& A \stackrel{\alpha}{\rightarrow} B \stackrel{\beta}{\rightarrow} C \stackrel{e_{C}}{\rightarrow} C \stackrel{e_{C}}{\rightarrow} C \rightarrow \varnothing .
\end{aligned}
$$

(Since, as can be easily seen, $\varnothing \rightarrow A \rightarrow{ }^{{ }} A A, C \rightarrow{ }^{\circ} C C \rightarrow \varnothing$ have property (E)). Thus $\operatorname{Im} \alpha=(A, \alpha)=\operatorname{Ker} \beta$.

From (1) we see that $\operatorname{Ker} \alpha=(\varnothing, 0)$ and from (2) we see that $\operatorname{Im} \beta=(C$, $\left.e_{C}\right)$. But it can be trivially verified that $\operatorname{Im}(\varnothing \rightarrow A)=(\varnothing, 0)$ and $\operatorname{Ker}(C \rightarrow \varnothing)$ $=\left(C, e_{C}\right)$. Thus, we have shown that $\varnothing \rightarrow A \rightarrow^{\alpha} B$ and $B \rightarrow^{\beta} C \rightarrow \varnothing$ are also exact, hence the necessity has been established.

For sufficiency, we assume that $\varnothing \rightarrow A \rightarrow^{\alpha} B, A \rightarrow^{\alpha} B \rightarrow^{\beta} C, B \rightarrow C \rightarrow \varnothing$ are exact. This tells us that $\operatorname{Im} \beta=\left(C, e_{C}\right), \operatorname{Ker} \alpha=(\varnothing, 0)$ and $\operatorname{Im} \alpha=\operatorname{Ker} \beta$. Hence we can write the decompositions of $\alpha$ and $\beta$ as

$$
\begin{array}{lr}
\varnothing \rightarrow A \stackrel{e_{A}}{\rightarrow} A \stackrel{\theta}{\rightarrow} D \stackrel{\kappa}{\rightarrow} B \stackrel{\pi}{\rightarrow} F, & \kappa \theta=\alpha, \\
D \stackrel{\kappa}{\rightarrow} B \stackrel{\pi^{\prime}}{\rightarrow} F^{\prime} \stackrel{\theta^{\prime}}{\rightarrow} C \stackrel{e_{C}}{\rightarrow} C \rightarrow \varnothing, & \theta^{\prime} \pi^{\prime}=\beta .
\end{array}
$$

Now we must verify conditions (1), (2) and (3) of property (E).

$$
\beta \alpha=\theta^{\prime} \pi^{\prime}{ }^{\prime} \theta=0 .
$$

Now, suppose $\alpha^{\prime}: A^{\prime} \rightarrow B$ and $\beta \alpha^{\prime}=0$. Then $\theta^{\prime} \pi^{\prime} \alpha^{\prime}=0$ implies $\pi^{\prime} \alpha^{\prime}=0$. Hence there is a unique $\gamma^{\prime}: A^{\prime} \rightarrow D$ such that $\kappa \gamma^{\prime}=\alpha^{\prime}$. But $\gamma=\theta^{-1} \gamma^{\prime}: A^{\prime} \rightarrow A$ and $\alpha\left(\theta^{-1} \gamma^{\prime}\right)=\kappa \theta \theta^{-1} \gamma^{\prime}=\kappa \gamma^{\prime}=\alpha^{\prime}$. If $\bar{\gamma}: A^{\prime} \rightarrow A$ and $\alpha \bar{\gamma}=\alpha^{\prime}$, then $\kappa \theta \bar{\gamma}=\kappa \gamma^{\prime}$ implies $\bar{\gamma}=\theta^{-1} \gamma^{\prime}$. Hence condition (2) is satisfied. Similarly one verifies (3) and this completes the proof.

Definitions. $\alpha: A \rightarrow B$ is a monomorphism if $\varnothing \rightarrow A \rightarrow{ }^{\alpha} B$ is exact. $\beta: B \rightarrow C$ is an epimorphism if $B \rightarrow{ }^{\beta} C \rightarrow \varnothing$ is exact.

3. G-graded categories. To cover the case when we are dealing with categories of graded modules over graded rings, we introduce the notion of a $G$-graded category $(\mathcal{A}, G)$ where $G$ is an abelian group. In most applications, $G$ is the group of integers $Z$, or a direct sum of $Z$ 's.

A $G$-graded category is given by the following four data:

(i) a collection of objects $\{A\}$;

(ii) a distinguished object $\varnothing$, called the zero object;

(iii) an abelian group $H(A, B)$ for any pair of objects $A, B \in(\mathcal{A}, G)$, an abelian group $H_{g}(A, B) \subset H(A, B)$ for any pair of objects $A, B \in(\mathcal{A}, G)$ and any $g \in G$. A map $\phi \in H_{o}(A, B)$ is said to be homogeneous of degree $g$, and we still write $\phi: A \rightarrow B$; 
(iv) a homomorphism $H_{g_{2}}(B, C) \otimes H_{\theta_{1}}(A, B) \rightarrow H_{\theta_{1}+o_{2}}(A, C)$ given for each triple of objects $A, B, C \in(\mathcal{A}, G)$ and each pair of elements $g_{1}, g_{2} \in G$.

Five axioms are imposed on these primitive terms.

Axıом 0. $H_{g_{1}}(A, B) \cap H_{g_{2}}(A, B)=0$ if $g_{1} \neq g_{2}$.

Axioms I-III are the same as those for an exact category, applied to homogeneous maps, Axiom III implying that $e_{A}$ has degree 0 .

Axгом IV is the same as Axiom IV for exact categories applied to homogeneous maps, except that $\sigma, \tau, \kappa, \pi$ have degree 0 , thereby implying that the degree of $\theta$ is the same as the degree of $\alpha$. (We assume property (E) defined only for homogeneous maps.)

An exact category is a special case of a $G$-graded category; $G$ is taken to be trivial and $H_{0}(A, B)=H(A, B)$. In the sequel, we may think of the theorems as being proved for $G$-graded categories. However, we shall suppress the degrees of the maps and in general use the language of exact categories.

4. Duality. For subsequent work, we shall have to deal with the dual category $\mathcal{A}^{*}$ of an exact category $\mathcal{A}$. We shall consider a metamathematical duality theorem afterwards.

The objects of $\mathcal{A}^{*}$ are symbols $A^{*}$ with $A \in \mathcal{A}$; the zero object of $\mathcal{A}^{*}$ is $\varnothing^{*}$; the group $H\left(A^{*}, B^{*}\right)$ is defined as $H(B, A)$ (for graded categories $\left.H_{o}\left(A^{*}, B^{*}\right)=H_{-o}(B, A)\right)$; for each map $\phi: B \rightarrow A$ in $A$ we denote by $\phi^{*}$ : $A^{*} \rightarrow B^{*}$ the corresponding "dual" map in $\mathcal{A}^{*}$; the composition in $\mathcal{A}^{*}$ is given by $\psi^{*} \phi^{*}=(\phi \psi)^{*}$.

$\mathcal{A}^{*}$ is clearly an exact category; Axioms I and II are trivial to verify. In Axiom III, define $e_{A *}$ to be $\left(e_{A}\right)^{*}$. To verify Axiom IV, we suppose we have $\alpha^{*}: A^{*} \rightarrow B^{*}$. Then $\alpha: B \rightarrow A$. We therefore have $K \rightarrow^{\sigma} B \rightarrow^{\tau} I \rightarrow^{0} I^{\prime} \rightarrow^{*} A \rightarrow^{\pi} F$. But then dualizing we obtain $F^{*} \rightarrow^{\pi *} A^{*} \rightarrow^{{ }^{*}} I^{* *} \rightarrow^{\theta *} I^{*} \rightarrow^{r *} B^{*} \rightarrow^{\sigma *} K^{*}$. Now $\tau^{*} \theta^{*} \kappa^{*}=(\kappa \theta \tau)^{*}=\alpha^{*}$. $\theta^{*}$ is an equivalence for $\left(\theta \theta^{-1}\right)^{*}=\left(e_{I^{\prime}}\right)^{*}=e_{I^{\prime} *}$, and $\left(\theta^{-1} \theta\right)^{*}=e_{I^{*}}$. It is obvious that $F^{*} \rightarrow A^{*} \rightarrow I^{*}$ and $I^{*} \rightarrow B^{*} \rightarrow K^{*}$ both have property (E).

Now for the metamathematical duality we replace the primitive term $H(A, B)$ by $H(B, A)$, i.e., we "reverse arrows." We also replace $H(B, C)$ $\otimes H(A, B) \rightarrow^{\xi A, B, C} H(A, C)$ by $H(B, A) \otimes H(C, B) \rightarrow^{\xi C, B, A} H(C, A)$.

Making these substitutions, we see that Axioms I-IV are unchanged. Hence we arrive at the

Duality Theorem 4.1. Let $S$ be a statement in $A, S^{*}$ the "dual" statement in $A$ (i.e., the statement obtained from $S$ by the above-mentioned substitutions). Then $S$ is true in $A$ if and only if $S^{*}$ is true in $\mathcal{A}$.

Throughout the rest of this paper, we shall state lemmas, but not their duals, it being understood that the latter are also true.

5. Fundamental lemmas.

Lemma 5.1. Let $\alpha: A \rightarrow B$ be a monomorphism. Then there is a mapping $\beta$ 
and an object $C$ such that $\varnothing \rightarrow A \rightarrow^{\alpha} B \rightarrow^{\beta} C \rightarrow \varnothing$ is exact. (For graded categories, $\beta$ can be chosen to be of degree zero.)

Lemma 5.2. $A$ necessary and sufficient condition that $\varnothing \rightarrow A \rightarrow^{\alpha} B \rightarrow^{\beta} C$ be exact is that $\beta \alpha=0$ and for every map $f: A^{\prime} \rightarrow B$ such that $\beta f=0$, there is a unique $f^{\prime}: A^{\prime} \rightarrow A$ such that $f=\alpha f^{\prime}$.

Lemma 5.3. A necessary and sufficient condition that $\alpha$ be a monomorphism is that if $f$ is any map such that $\alpha f=0$, then $f=0$.

Theorem 5.4. A necessary and sufficient condition that $\varnothing \rightarrow A \rightarrow B \rightarrow \varnothing$ be exact is that $\theta$ be an equivalence.

TheOREM 5.5. Let

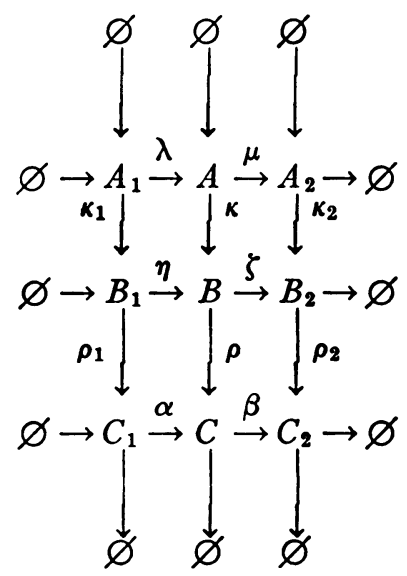

be a commutative diagram with exact columns. If the bottom two rows are exact, then so is the top row.

Proof. First we show that $\mu \lambda=0 . \kappa_{2} \mu \lambda=\zeta \eta \kappa_{1}=0$. Thus $\mu \lambda=0$.

Next we see that $\lambda$ is a monomorphism. Suppose $\lambda f=0$. Then $\kappa \lambda f=0$ $=\eta \kappa_{1} f$, which implies $f=0$. Therefore, we can find a map $\omega$ and an object $N$ such that $\varnothing \rightarrow A_{1} \rightarrow^{\lambda} A \rightarrow^{\omega} N \rightarrow \varnothing$ is exact. We must now show that $N$ is equivalent to $A_{2}$, i.e., we must produce an equivalence $\theta: N \rightarrow A_{2}$ such that $\theta \omega=\mu$. To do this, we apply the first Noether isomorphism theorem (which holds in an exact category $A$ ), to the following sets of exact sequences:

$$
\begin{aligned}
& \varnothing \rightarrow B_{1} \stackrel{\eta}{\rightarrow} B \stackrel{\zeta}{\rightarrow} B_{2} \rightarrow \varnothing, \\
& \varnothing \rightarrow A_{1} \stackrel{\kappa_{1}}{\rightarrow} B_{1} \stackrel{\rho_{1}}{\rightarrow} C_{1} \longrightarrow \varnothing, \\
& \varnothing \rightarrow A_{1} \stackrel{\eta \kappa_{1}}{\rightarrow} B \stackrel{\tau}{\rightarrow} F\left(B, A_{1}\right) \rightarrow \varnothing,
\end{aligned}
$$




$$
\varnothing \rightarrow A \stackrel{\kappa}{\rightarrow} B \stackrel{p}{\rightarrow} C \longrightarrow \varnothing
$$

$$
\begin{aligned}
& \varnothing \rightarrow A_{1} \stackrel{\lambda}{\rightarrow} A \stackrel{\omega}{\rightarrow} N \longrightarrow \varnothing, \\
& \varnothing \rightarrow A_{1} \stackrel{\eta \kappa_{1}}{\rightarrow} B \stackrel{\tau}{\rightarrow} F\left(B, A_{1}\right) \rightarrow \varnothing,
\end{aligned}
$$

and obtain

$$
\varnothing \rightarrow C_{1} \stackrel{n_{1}}{\rightarrow} F\left(B, A_{1}\right) \stackrel{m_{1}}{\rightarrow} B_{2} \rightarrow \varnothing,
$$

exact with $n_{1} \rho_{1}=\tau \eta, m_{1} \tau=\zeta$;

$$
\varnothing \rightarrow N \stackrel{n_{2}}{\rightarrow} F\left(B, A_{1}\right) \stackrel{m_{2}}{\rightarrow} C \rightarrow \varnothing
$$

exact with $n_{2} \omega=\tau \kappa, m_{2} \tau=\rho$.

We then apply the dual to the Noether isomorphism theorem to

$$
\varnothing \rightarrow N \stackrel{n_{2}}{\rightarrow} F\left(B, A_{1}\right) \stackrel{m_{2}}{\rightarrow} C \rightarrow \varnothing
$$

$$
\begin{aligned}
& \varnothing \rightarrow C_{1} \stackrel{\alpha}{\rightarrow} C \stackrel{\beta}{\rightarrow} C_{2} \rightarrow \varnothing, \\
& \varnothing \rightarrow R \stackrel{\sigma}{\rightarrow} F\left(B, A_{1}\right) \stackrel{\beta m_{2}}{\rightarrow} C_{2} \rightarrow \varnothing, \\
& \varnothing \rightarrow C_{1} \stackrel{n_{1}}{\rightarrow} F\left(B, A_{1}\right) \stackrel{m_{1}}{\rightarrow} B_{2} \rightarrow \varnothing,
\end{aligned}
$$

$$
\begin{aligned}
& \varnothing \rightarrow A_{2} \stackrel{\kappa_{2}}{\rightarrow} B_{2} \stackrel{\rho_{2}}{\rightarrow} C_{2} \rightarrow \varnothing, \\
& \varnothing \rightarrow R \stackrel{\sigma}{\rightarrow} F\left(B, A_{1}\right) \stackrel{\beta m_{2}}{\rightarrow} C_{2} \rightarrow \varnothing,
\end{aligned}
$$

and obtain

$$
\varnothing \rightarrow N \stackrel{n_{3}}{\rightarrow} R \stackrel{m_{3}}{\rightarrow} C_{1} \rightarrow \varnothing
$$

exact with $\sigma n_{8}=n_{2}, \alpha m_{3}=m_{2} \sigma$;

$$
\varnothing \rightarrow C_{1} \stackrel{n_{4}}{\rightarrow} R \stackrel{m_{4}}{\rightarrow} A_{2} \rightarrow \varnothing
$$

exact with $\sigma n_{4}=n_{1}, \kappa_{2} m_{4}=m_{1} \sigma$.

Now we can show that $m_{3} n_{4}=e_{C_{1}} ; \alpha m_{3} n_{4}=m_{2} \sigma n_{4}=m_{2} n_{1}, m_{2} n_{1} \rho_{1}=m_{2} \tau \eta$ $=\rho \eta=\alpha \rho_{1}$ which implies $m_{2} n_{1}=\alpha . \alpha m_{3} n_{4}=\alpha$ implies $m_{3} n_{4}=e_{c_{1}}$.

Let $\theta=m_{1} n_{3}: N \rightarrow A_{2}$. Then $\theta \omega=\mu$ for $\kappa_{2} \theta \omega=\kappa_{2} m_{1} n_{3} \omega=m_{1} \sigma n_{3} \omega=m_{1} n_{2} \omega$ $=m_{1} \tau \kappa=\zeta \kappa=\kappa_{2} \mu$. Hence $\theta \omega=\mu$. Now we show that $\theta$ is an equivalence.

Let $f \theta=0$. Then $\left(f m_{4}\right) n_{3}=0$ implies that $f m_{4}=f^{\prime} m_{3}$. Since $0=f m_{4} n_{4}$ $=f^{\prime} m_{3} n_{4}=f^{\prime}$, we see that $f m_{4}=0$, hence $f=0$. If $\theta g=0$, then $m_{4}\left(n_{3} g\right)=0$ implies that $n_{3} g=n_{4} g^{\prime}$. Since $0=m_{3} n_{3} g=m_{3} n_{4} g^{\prime}=g^{\prime}$, we have $n_{8} g=0$, hence 
$g=0$. Thus $\theta$ is both a monomorphism and an epimorphism, hence an equivalence.

We remark here that this proof shows that $R$ is the direct sum of $A_{2}$ and $C_{1}$ (see Part III, §1, Remark).

LEMMA 5.6. Let

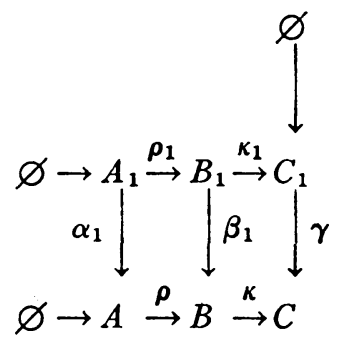

be a commutative diagram with exact rows and columns. Then

(a) $\operatorname{Ker} \alpha_{1} \approx \operatorname{Ker} \beta_{1}$,

(b) There is a monomorphism $\mu: \operatorname{Im} \alpha_{1} \rightarrow \operatorname{Im} \beta_{1}$,

(c) There is a monomorphism $\iota$ : Coker $\rho_{1} \rightarrow \operatorname{Coker} \rho$,

(d) Coker $\rho_{1} \approx$ Coker $\mu$.

Lemma 5.7. If $A_{1} \rightarrow^{\alpha_{1}} A \rightarrow^{\alpha_{3}} A_{2}$ can be imbedded in the commutative diagram

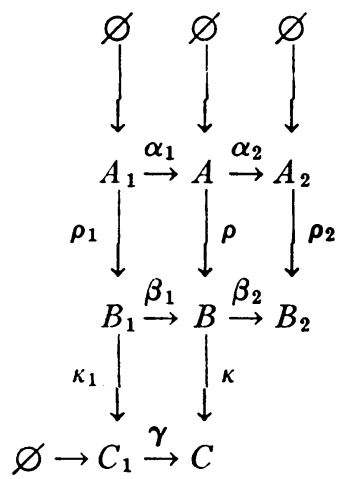

in which the columns and bottom two rows are exact, then $A_{1} \rightarrow^{\alpha_{1}} A \rightarrow^{\alpha_{2}} A_{2}$ is exact.

TheOREM 5.8. Consider the commutative diagram

$(\mathrm{H})$

$$
\begin{aligned}
& Z_{1}^{\prime} \stackrel{\alpha_{1}}{\rightarrow} Z^{\prime} \stackrel{\alpha_{2}}{\rightarrow} Z_{2}^{\prime} \rightarrow \varnothing \\
& d_{1}|d| d_{2} d_{\beta_{2}} \\
& \varnothing \rightarrow Z_{1} \stackrel{\beta_{1}}{\rightarrow} Z \stackrel{\beta_{2}}{\rightarrow} Z_{2}
\end{aligned}
$$

with exact rows. Then 
(a) Ker $d_{1} \rightarrow \operatorname{Ker} d \rightarrow \operatorname{Ker} d_{2}$ is exact,

(b) Coker $d_{1} \rightarrow$ Coker $d \rightarrow$ Coker $d_{2}$ is exact,

(c) There is a map $\mathcal{L}$ : Ker $d_{2} \rightarrow$ Coker $d_{1}$ such that

$$
\operatorname{Ker} d \rightarrow \operatorname{Ker} d_{2} \stackrel{\mathcal{L}}{\rightarrow} \text { Coker } d_{1} \rightarrow \text { Coker } d \text { is exact. }
$$

Proof. (a) and (b) are trivial consequences of Lemma 5.7 (and its dual). Therefore, we need only concern ourselves with (c). We shall use two dual constructions of the map $\mathcal{L}$, obtaining a map $\mathcal{L}_{1}$ and a map $\mathcal{L}_{2}$, and show that $\mathcal{L}_{1}=\mathcal{L}_{2}$. This will demonstrate the self-duality of $\mathcal{L}$.

We first employ a procedure which is tantamount to taking the counterimage. Consider the commutative diagram

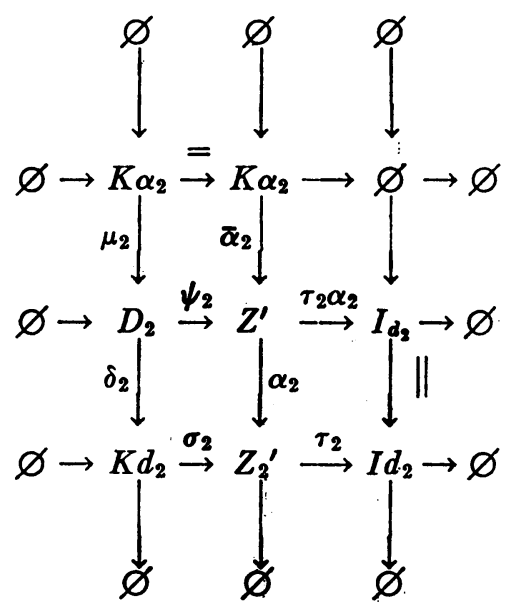

The three rows and the middle and right columns are exact by definition; $\mu_{2}$ and $\delta_{2}$ are naturally induced maps and $D_{2}=\operatorname{Ker} \tau_{2} \alpha_{2}$. Thus the diagram is commutative and the left column is exact. Since $\beta_{2} d \psi_{2}=d_{2} \alpha_{2} \psi_{2}=d_{2} \sigma_{2} \delta_{2}=0$, we know that $d \psi_{2}=\beta_{1} \iota_{2}$ where $\iota_{2}: D_{2} \rightarrow Z_{1}$. Now we examine the following exact sequences

$$
\begin{aligned}
& \varnothing \rightarrow K_{d_{1}} \stackrel{\sigma_{1}}{\rightarrow} Z_{1}^{\prime} \stackrel{\tau_{1}}{\rightarrow} I_{d_{1}} \rightarrow \varnothing, \\
& \varnothing \rightarrow I_{d_{1}} \stackrel{\kappa_{1}}{\rightarrow} Z_{1} \stackrel{\pi_{1}}{\rightarrow} F_{1} \rightarrow \varnothing, \\
& \varnothing \rightarrow K \alpha_{1} \stackrel{\boldsymbol{\alpha}_{1}}{\rightarrow} Z_{1}^{\prime} \stackrel{\tilde{\pi}_{1}}{\rightarrow} K \alpha_{2} \rightarrow \varnothing,
\end{aligned}
$$

where $\kappa_{1} \tau_{1}=d_{1}$ and the last sequence is exact due to the exactness of the top row of $(\mathrm{H})$. Also $\bar{\alpha}_{2} \bar{\pi}_{1}=\alpha_{1}$.

Now $\tau_{1} \bar{\alpha}_{1}=0$ for $\beta_{1} \kappa_{1} \tau_{1} \bar{\alpha}_{1}=\beta_{1} d_{1} \bar{\alpha}_{1}=d \alpha_{1} \bar{\alpha}_{1}=0$. Therefore $\bar{\alpha}_{1}=\sigma_{1} \xi_{2}$ where $\xi_{2}: K \alpha_{1} \rightarrow K_{d_{1}}$. $\xi_{2}$ is, of course, a monomorphism. Thus we have the commutative diagram 


$$
\xi_{\xi_{2}} K \alpha_{1} \stackrel{\alpha_{1}}{\rightarrow} Z_{1}^{\prime} \stackrel{\sigma_{1}}{\rightarrow} K \alpha_{1} \rightarrow \stackrel{\tau_{1}}{\rightarrow} I_{d_{1}} \rightarrow \varnothing
$$

and therefore an epimorphism $\eta_{2}: K \alpha_{2} \rightarrow I_{d_{1}}$ such that $\eta_{2} \bar{\pi}_{1}=\tau_{1}$. We observe the following equalities:

$$
\begin{aligned}
& \beta_{1} \kappa_{1} \eta_{2} \bar{\pi}_{1}=\beta_{1} \kappa_{1} \tau_{1}=\beta_{1} d_{1}=d \alpha_{1}, \\
& \beta_{1} \iota_{2} \mu_{2} \bar{\pi}_{1}=d \psi_{2} \mu_{2} \bar{\pi}_{1}=d \bar{\alpha}_{2} \bar{\pi}_{1}=d \alpha_{1} .
\end{aligned}
$$

Hence $\kappa_{1} \eta_{2}=\iota_{2} \mu_{2}$ so we obtain the commutative diagram

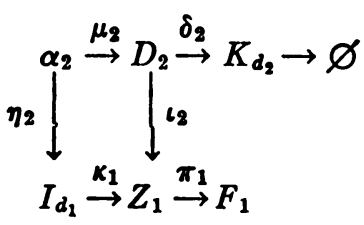

and obtain the map $\mathcal{L}_{2}: K_{d_{2}} \rightarrow F_{1}$ with $\mathcal{L}_{2} \delta_{2}=\pi_{1} \iota_{2}$.

Construction of $\mathcal{L}_{1}$. In this construction we shall only label the diagrams and maps and not bother with the verification of each step.

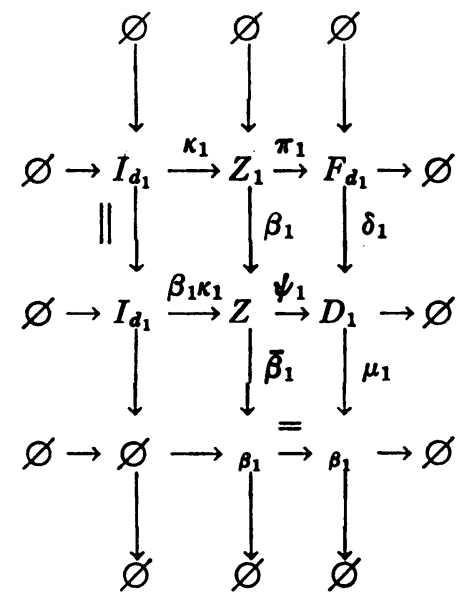

$\psi_{1} d \alpha_{1}=0$ so $\psi_{1} d=\iota_{1} \alpha_{2}$ where $\iota_{1}: Z_{2}^{\prime} \rightarrow D_{1}$. Then we have

$$
\begin{aligned}
& \varnothing \rightarrow F_{\beta_{1}} \stackrel{\bar{\sigma}_{2}}{\rightarrow} Z_{2} \stackrel{\bar{\beta}_{2}}{\rightarrow} F_{\beta_{2}} \rightarrow \varnothing, \\
& \varnothing \rightarrow K_{d_{2}} \stackrel{\sigma_{2}}{\rightarrow} Z_{2}^{\prime} \stackrel{\tau_{2}}{\rightarrow} I_{d_{2}} \rightarrow \varnothing, \\
& \varnothing \rightarrow I_{d_{2}} \stackrel{\kappa_{2}}{\rightarrow} Z_{2} \stackrel{\pi_{2}}{\rightarrow} F_{d_{2}} \rightarrow \varnothing,
\end{aligned}
$$




$$
\begin{array}{rlrl}
\kappa_{2} \tau_{2} & =d_{2}, & & \bar{\sigma}_{2} \bar{\beta}_{1}=\beta_{2}, \\
\bar{\beta}_{2} & =\xi_{1} \pi_{2}, & & \xi_{1}: F d_{2} \rightarrow F \beta_{2}, \\
\kappa_{2}=\bar{\sigma}_{2} \eta_{1}, & & \eta_{1}: I d_{2} \rightarrow F \beta_{1},
\end{array}
$$

and $\eta_{1} \tau_{2}=\mu_{1} \iota_{1}$. Therefore we have a map $\mathcal{L}_{1}: K_{d_{2}} \rightarrow F_{d_{1}}$ such that $\delta_{1} \mathcal{L}_{1}=\iota_{1} \sigma_{2}$. To see that $\mathcal{L}_{1}=\mathcal{L}_{2}$, we observe that

$$
\delta_{1} \mathcal{L}_{1} \delta_{2}=\iota_{1} \sigma_{2} \delta_{2}=\iota_{1} \alpha_{2} \psi_{2}=\psi_{1} d \psi_{2}=\psi_{1} \beta_{1 \iota_{2}}=\delta_{1} \pi_{1} \iota_{2}=\delta_{1} \mathcal{L}_{2} \delta_{2} \text {. }
$$

In a $G$-graded category, we observe that $\mathcal{L}_{1}, \mathcal{L}_{2}$ can be constructed to have the same degree as $d$, provided $\alpha_{1}, \alpha_{2}, \beta_{1}, \beta_{2}$ have degree zero.

We define $\mathcal{L}=\mathcal{L}_{1}=\mathcal{L}_{2}: K_{d_{3}} \rightarrow F_{d_{1}}$.

It will now suffice to show that $\operatorname{Ker} d \rightarrow \omega_{2} \operatorname{Ker} d_{2} \rightarrow \mathcal{L}$ Coker $d_{1}$ is exact, for the proof of the other exactness is dual to this. We record the fact that $\alpha_{2} \sigma=\sigma_{2} \omega_{2}$ where $\sigma: \operatorname{Ker} d \rightarrow Z^{\prime}$.

$$
\delta_{1} \mathcal{L} \omega_{2}=\iota_{1} \sigma_{2} \omega_{2}=\iota_{1} \alpha_{2} \sigma=\psi_{1} d \sigma=0 .
$$

Hence $\mathcal{L} \omega_{2}=0$. We have

$$
\begin{aligned}
& \varnothing \rightarrow K \omega_{2} \stackrel{\rho_{1}}{\rightarrow} \operatorname{Ker} d \stackrel{\phi_{1}}{\rightarrow} I \omega_{2} \rightarrow \varnothing, \\
& \varnothing \rightarrow I \omega_{2} \stackrel{\lambda_{1}}{\rightarrow} \operatorname{Ker} d_{2} \stackrel{\nu_{1}}{\rightarrow} F \omega_{2} \rightarrow \varnothing, \\
& \varnothing \rightarrow K \mathcal{L} \stackrel{\rho_{2}}{\rightarrow} \operatorname{Ker} d_{2} \stackrel{\phi_{2}}{\rightarrow} I \mathcal{L} \rightarrow \varnothing, \\
& \varnothing \rightarrow I \mathcal{L} \stackrel{\lambda_{2}}{\rightarrow} \operatorname{Coker} d_{1} \stackrel{\nu_{2}}{\rightarrow} F \mathcal{L} \rightarrow \varnothing,
\end{aligned}
$$

exact with $\lambda_{1} \phi_{1}=\omega_{2}, \lambda_{2} \phi_{2}=\mathcal{L}$. Now $\phi_{2} \lambda_{1}=0$ since $\lambda_{2} \phi_{2} \lambda_{1} \phi_{1}=\mathcal{L} \omega_{2}=0$. Thus we have $\theta: I \omega_{2} \rightarrow K \mathcal{K}$ with $\rho_{2} \theta=\lambda_{1} . \theta$ is a monomorphism.

We must show that $\theta$ is also an epimorphism. To this end, consider the commutative diagram

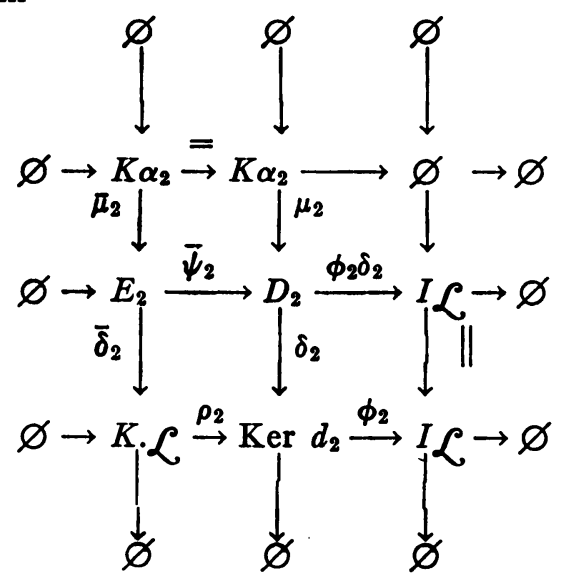


with exact rows and columns. Since $\pi_{1} \iota_{2} \bar{\psi}_{2}=\mathcal{L} \delta_{2} \bar{\psi}_{2}=\mathcal{L} \rho_{2} \bar{\delta}_{2}=0$, we have $\iota_{2} \Psi_{2}=\kappa_{1} u$ where $u: E_{2} \rightarrow I d_{1}$. We claim that $u$ is an epimorphism, for $\kappa_{1} u \bar{\mu}_{2}$ $=\iota_{2} \bar{\psi}_{2} \bar{\mu}_{2}=\iota_{2} \mu_{2}=\kappa_{1} \eta_{2}$ implies that $u \bar{\mu}_{2}=\eta_{2}$. We shall establish a map $\bar{r}: \operatorname{Ker} d$ $\rightarrow E_{2}$ and prove that $\varnothing \rightarrow \operatorname{Ker} d \rightarrow^{\bar{r}} E_{2} \rightarrow^{\mathbf{x}} I d_{1} \rightarrow \varnothing$ is exact.

Since $\tau_{2} \alpha_{2} \sigma=0, \sigma=\psi_{2} r$ where $r: \operatorname{Ker} d \rightarrow D_{2}$. But $\delta_{1} \lambda_{2} \phi_{2} \delta_{2} r=\psi_{1} d \psi_{2} r=\psi_{1} d \sigma=0$, hence $\phi_{2} \delta_{2} r=0$ and $r=\bar{\psi}_{2} \bar{r}$. Now consider

$$
\varnothing \rightarrow K u \stackrel{\sigma_{u}}{\rightarrow} E_{2} \stackrel{u}{\rightarrow} I d_{1} \rightarrow \varnothing
$$

exact. $\beta_{1} \kappa_{1} u \bar{r}=\beta_{1} \iota_{2} \bar{\psi}_{2} \bar{r}=d \psi_{2} r=d \sigma=0$, hence $u \bar{r}=0$ and $\bar{r}=\sigma_{u} \Omega$ where $\Omega$ : Ker $d$ $\rightarrow K u$. Now we have

$$
\begin{aligned}
& \varnothing \rightarrow \operatorname{Ker} \stackrel{\stackrel{\vec{r}}{\rightarrow}}{\rightarrow} E_{2} \stackrel{v}{\rightarrow} F_{\bar{r}} \rightarrow \varnothing, \\
& \varnothing \rightarrow E_{2} \stackrel{\psi_{2} \bar{\psi}_{2}}{\rightarrow} Z^{\prime} \stackrel{s}{\rightarrow} F \psi_{2} \Psi_{2} \rightarrow \varnothing, \\
& \varnothing \rightarrow \operatorname{Ker} d \stackrel{\sigma}{\rightarrow} Z^{\prime} \stackrel{\tau}{\rightarrow} I d \rightarrow \varnothing,
\end{aligned}
$$

exact with $\sigma=\psi_{2} \Psi_{2} \bar{r}$, hence

$$
\varnothing \rightarrow F_{\bar{r}} \stackrel{n}{\rightarrow} I d \stackrel{m}{\rightarrow} F \psi_{2} \psi_{2} \rightarrow \varnothing
$$

is exact with $n v=\tau \psi_{2} \bar{\psi}_{2}$.

$$
\kappa n v \sigma_{u}=\kappa \tau \psi_{2} \bar{\psi}_{2} \sigma_{u}=d \psi_{2} \bar{\psi}_{2} \sigma_{u}=\beta_{1 \iota_{2}} \bar{\psi}_{2} \sigma_{u}=\beta_{1 \kappa_{1}} u \sigma_{u}=0
$$

implies that $v \sigma_{u}=0$, and we obtain $\Omega^{\prime}: K u \rightarrow \operatorname{Ker} d$ with $\bar{r} \Omega^{\prime}=\sigma_{u}$. It is trivial to show that $\Omega^{\prime}=\Omega^{-1}$. Thus our assertion that $\varnothing \rightarrow \operatorname{Ker} d \rightarrow^{\bar{r}} \dot{E}_{2} \rightarrow^{u} I d_{1} \rightarrow \varnothing$ is exact is proved.

We next study the commutative diagram

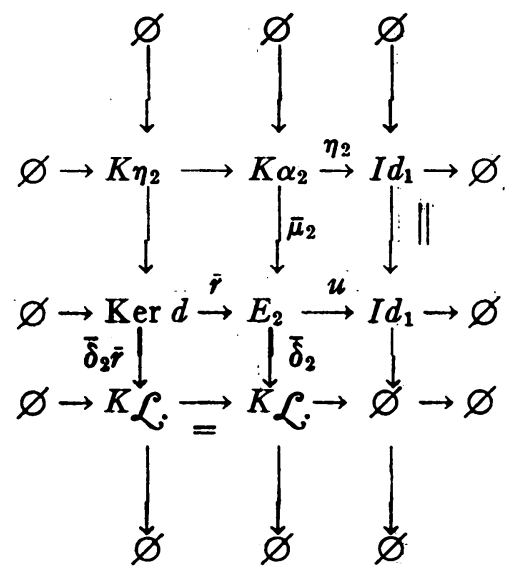


and deduce that the left column is exact, in particular, that $\bar{\delta}_{2} \bar{r}$ is an epimorphism. We show that $\theta \phi_{1}=\bar{\delta}_{2} \bar{r}$ :

$$
\sigma_{2} \rho_{2} \theta \phi_{1}=\sigma_{2} \lambda_{1} \phi_{1}=\sigma_{2} \omega_{2}=\alpha_{2} \sigma=\alpha_{2} \psi_{2} r=\sigma_{2} \delta_{2} \bar{\psi}_{2} \bar{r}=\sigma_{2} \rho_{2} \bar{\delta}_{2} \bar{r}_{\text {. }}
$$

Hence $\theta \phi_{1}=\bar{\delta}_{2} \bar{r}$ and $\theta$ is thereby an epimorphism.

For the remaining lemmas, we shall make use of the following diagram

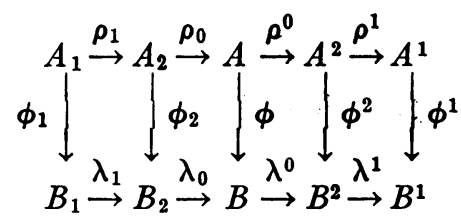

which is commutative and has exact rows.

Theorem 5.9 (Five Lemma). Let $\phi_{1}$ be an epimorphism, $\phi^{2}$ a monomorphism. Then $\operatorname{Ker} \phi \approx \operatorname{Im} \rho_{0} \sigma\left(\phi_{2}\right)$ where $\sigma\left(\phi_{2}\right): \operatorname{Ker} \phi_{2} \rightarrow A_{2}$.

Corollary 5.10. Let $\phi_{1}$ be an epimorphism, $\phi_{2}$ and $\phi^{2}$ monomorphisms. Then $\phi$ is a monomorphism.

CoROllary 5.11. Let $\phi_{1}$ be an epimorphism, $\phi^{1}$ a monomorphism, and let $\phi_{2}$ and $\phi^{2}$ be equivalences. Then $\phi$ is an equivalence.

\section{Part II. Homology in Graded Categories}

1. Abstract homology. Throughout this section, we shall be working with objects and maps in a fixed $G$-graded category. The degree of a mapping will take on more significance here than in the last chapter, and will be given more consideration. Henceforth, we shall write $A$ for $(\mathcal{A}, G)$.

Definition. A pair $(A, d), A \in \mathcal{A}, d \in H_{o}(A, A)$, will be called a $g$-derivation or an object with differentiation if $d^{2}=0$.

Let us now consider a $g$-derivation $(A, d)$ and the consequent factorization of $A \rightarrow^{d} A$ :

$$
\begin{aligned}
& \varnothing \rightarrow Z \stackrel{\sigma}{\rightarrow} A \stackrel{\tau}{\rightarrow} B \rightarrow \varnothing \\
& B \stackrel{\theta}{\rightarrow} B^{\prime} \\
& \varnothing \rightarrow B^{\prime} \stackrel{\boldsymbol{k}}{\rightarrow} A \stackrel{\boldsymbol{\pi}}{\rightarrow} Z^{\prime} \rightarrow \varnothing .
\end{aligned}
$$

We recall that $\sigma, \tau, \kappa, \pi$ are of degree zero, and $\theta$ is of degree $g$. We must also keep in mind the fact that this factorization is unique only up to a transitive family of equivalences of degree zero (viz. Part I, 1.2).

We can obtain a unique map $\omega: B^{\prime} \rightarrow Z$ of degree zero such that $\sigma \omega=\kappa$ for $(\kappa \theta)(\tau \kappa)(\theta \tau)=(\kappa \theta \tau)(\kappa \theta \tau)=d^{2}=0$ implies $\tau \kappa=0$.

Similarly, we can obtain a unique map $\phi: Z^{\prime} \rightarrow B$ of degree zero such that 
$\phi \pi=\tau$. Also, we have the map $\pi \sigma: Z \rightarrow Z^{\prime}$ of degree zero, and so we have the sequence

$$
\varnothing \rightarrow B^{\prime} \stackrel{\omega}{\rightarrow} Z \stackrel{\pi \sigma}{\rightarrow} Z^{\prime} \stackrel{\phi}{\rightarrow} B \rightarrow \varnothing .
$$

Lemma 1.1. The sequence (1) is exact.

Since $\pi \sigma$ is of degree zero, we may choose as its canonical factorization

$$
\begin{aligned}
& \varnothing \rightarrow B^{\prime} \stackrel{\omega}{\rightarrow} Z \stackrel{\lambda}{\rightarrow} H \rightarrow \varnothing, \\
& \varnothing \rightarrow H \stackrel{\zeta}{\rightarrow} Z^{\prime} \stackrel{\phi}{\rightarrow} B \rightarrow \varnothing,
\end{aligned}
$$$$
\zeta \lambda=\pi \sigma .
$$

Let us consider the map $D=\omega \theta \phi: Z^{\prime} \rightarrow Z$. $D$ is a mapping of degree $g$. It is quite clear that

$$
\text { Ker } D=H=\text { Coker } D \text { or }
$$

$$
\varnothing \rightarrow B \stackrel{\zeta}{\rightarrow} Z^{\prime} \stackrel{D}{\rightarrow} Z \stackrel{\lambda}{\rightarrow} H \rightarrow \varnothing \text { is exact. }
$$

Definition. The pair $\left(Z, Z^{\prime}\right)$ is called the cycles of $(A, d)$, denoted sometimes by $Z(A), Z^{\prime}(A)$. The pair $\left(B, B^{\prime}\right)$ is called the boundaries of $(A, d)$, denoted by $B(A), B^{\prime}(A)$. The object $H$ will be called the homology of $(A, d)$, denoted by $H(A)$.

When there is no ambiguity, we shall write $Z$ instead of $Z(A)$, etc.

The self-duality of the definition of $H(A)$ may be stated in terms of the dual object $\left(A^{*}, d^{*}\right)$ in the category $A^{*}$. We have

$$
\begin{array}{cr}
Z\left(A^{*}\right)=\left[Z^{\prime}(A)\right]^{*}, & B\left(A^{*}\right)=\left[B^{\prime}(A)\right]^{*}, \\
Z^{\prime}\left(A^{*}\right)=[Z(A)]^{*}, & B^{\prime}\left(A^{*}\right)=[B(A)]^{*}, \\
H\left(A^{*}\right)=[H(A)]^{*} .
\end{array}
$$

Definition. A mapping $f:(A, d) \rightarrow(\bar{A}, \bar{d})$ of $g$-derivations is a mapping $f \in H_{h}(A, \bar{A})$ such that $f d=\bar{d} f$.

It will suffice to consider mappings of degree zero. There is nothing to be gained by treating the more general case.

Let $f:(A, d) \rightarrow(\bar{A}, d)$ be a mapping. Then we have the commutative diagrams

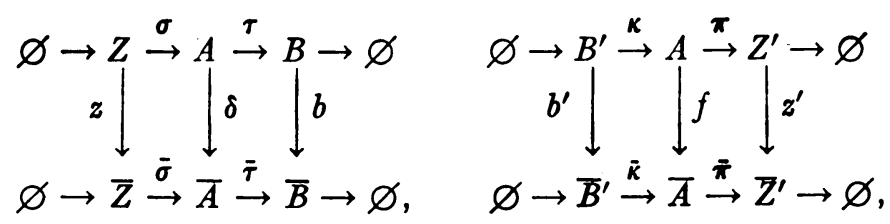




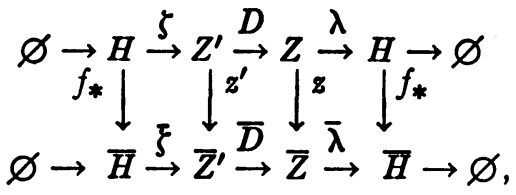

$$
\begin{aligned}
& \left.\stackrel{b}{i=} \stackrel{\theta}{\rightarrow}\right|_{\bar{B}} ^{B^{\prime}} b^{\prime}
\end{aligned}
$$

Definition. We shall say that the sequence of $g$-derivations

$$
\varnothing \rightarrow\left(A_{1}, d_{1}\right) \stackrel{\alpha}{\rightarrow}(A, d) \stackrel{\beta}{\rightarrow}\left(A_{2}, d_{2}\right) \rightarrow \varnothing
$$

is exact if

$$
\varnothing \rightarrow A_{1} \stackrel{\alpha}{\rightarrow} A \stackrel{\beta}{\rightarrow} A_{2} \rightarrow \varnothing
$$

is exact. ( $\alpha$ and $\beta$ are assumed to be maps of derivations.)

ThEOREM 1.2. Let $\varnothing \rightarrow\left(A_{1}, d_{1}\right) \rightarrow^{\alpha}(A, d) \rightarrow^{\beta}\left(A_{2}, d_{2}\right) \rightarrow \varnothing$ be an exact sequence of $\mathrm{g}$-derivations. Then there exists a map $\partial: H_{2} \rightarrow H_{1}$ such that the sequence

$$
H_{1} \stackrel{\alpha_{*}}{\rightarrow} H \stackrel{\beta_{*}}{\rightarrow} H_{2} \stackrel{\partial}{\rightarrow} H_{1} \stackrel{\alpha_{*}}{\rightarrow} H \stackrel{\beta_{*}}{\rightarrow} H_{2}
$$

is exact. The map $\partial$ has degree $g$.

Proof. This follows from Part I, 5.8 and the obvious exactness of $Z_{1}^{\prime} \rightarrow{ }^{\prime} Z^{\prime}$ $\rightarrow z_{2}^{\prime} Z_{2}^{\prime} \rightarrow \varnothing$ and $\varnothing \rightarrow Z_{1} \rightarrow{ }_{1}^{2} Z \rightarrow{ }_{2}^{2} Z_{2}$.

The usual propositions about homology can now be proved easily. However, for the sake of completeness, we make the following

Definition. $f, g:(A, d) \rightarrow(\bar{A}, \bar{d})$ are chain homotopic, $f \simeq g$, if there exists a mapping $s: A \rightarrow \bar{A}$ of degree minus (degree of $d$ ) such that

$$
\bar{d} s+s d=f-g \text {. }
$$

It is well known that chain homotopy is an equivalence relation.

\section{Complezes.}

Definition. A complex in $\mathcal{A}$ is a sequence

$$
A: \cdots \rightarrow A^{n-1} \stackrel{d^{n-1}}{\longrightarrow} A^{n} \stackrel{d^{n}}{\rightarrow} A^{n+1} \rightarrow \cdots
$$

with $d^{n} d^{n-1}=0$. (We are assuming, now, that we are in an exact category. Otherwise we would require that the degrees of all the $d^{k}$ be zero.)

As usual, we may define $Z^{n}=\operatorname{Ker} d^{n}, B^{n}=\operatorname{Im} d^{n-1}, B^{\prime n}=\operatorname{Coim} d^{n-1}$, $Z^{\prime n}=$ Coker $d^{n}$. 
For each $n$, we have the exact sequence

$$
\varnothing \rightarrow B^{\prime n} \stackrel{\omega^{n}}{\rightarrow} Z^{n} \stackrel{\pi^{n} \sigma^{n}}{\rightarrow} Z^{\prime n} \stackrel{\phi^{n}}{\rightarrow} B^{n} \rightarrow \varnothing
$$

and $H^{n}=\operatorname{Coker} \omega^{n}=\operatorname{Ker} \phi^{n}$.

Definition. A mapping $f: A \rightarrow \bar{A}$ of one complex into another is a sequence of maps $\left\{f^{n}\right\}$ where $f^{n}: A^{n} \rightarrow \bar{A}^{n}$ and $\bar{d}^{n} f^{n}=f^{n+1} d^{n}$.

The usual formal facts about homology for complexes may be obtained here.

3. Construction of graded categories. In this section, we shall give two constructions of categories. The first will correspond to the category of objects with differentiation $d(\mathcal{A}, G)$ in a $g$-graded category $(\mathcal{A}, G)$; the second will be the construction of a $g$-graded category starting with an exact category $A$ and an abelian group $G$; this will correspond to the category of complexes over an exact category.

Let $(\mathcal{A}, G)$ be a $G$-graded category. We define the objects of $d(\mathcal{A}, G)$ to be pairs $(A, d)$ where $(A, d)$ is a $g$-derivation. The zero object is $(\varnothing, 0)$. $H_{h}\left(\left(A_{1}, d_{1}\right),\left(A_{2}, d_{2}\right)\right)$ is the group of all maps $f:\left(A_{1}, d_{1}\right) \rightarrow\left(A_{2}, d_{2}\right)$ of degree $h$ and $H_{h_{2}}\left(\left(A_{2}, d_{2}\right),\left(A_{3}, d_{3}\right)\right) \otimes H_{h_{1}}\left(\left(A_{1}, d_{1}\right),\left(A_{2}, d_{2}\right)\right) \rightarrow H_{h_{1}+h_{2}}\left(\left(A_{1}, d_{1}\right),\left(A_{3}, d_{3}\right)\right)$ is the usual composition of maps. It is easy to see that $d(\mathcal{A}, G)$ is a $G$-graded category.

This construction essentially parallels the construction of the category of modules over the ring of dual numbers $\Gamma=(\Lambda, d)$ obtained from the category of modules over the ring $\Lambda$.

For our second construction, we let $\mathcal{A}$ be an exact category, $G$ an abelian group. Our objects are defined to be functions $A$ from $G$ to the objects of $\mathcal{A}$. The zero object, $\varnothing$, is the function $\varnothing(g)=\varnothing$ all $g \in G$. For each $g^{\prime} \in G$, and each pair of objects $A, B$, we consider functions $\alpha$ defined on $G$ such that $\alpha(g) \in H\left(A(g), B\left(g+g^{\prime}\right)\right)$. We define addition in the obvious way and thus turn the set of such $\alpha^{\prime}$ 's into an abelian group $H_{g^{\prime}}(A, B)$. The composition of two such maps is defined in the natural way.

It is easy to verify that we again have a $G$-graded category.

The usual case occurs when $g$ is the group $Z$ of integers. In that case, the 1-derivations are precisely the complexes of $\$ 2$.

4. Functors and homology. Throughout this paper, by the term "functor" we shall mean an additive functor [1]. We are varying from the standard use of the term here [2] because of the additional additive structure of an exact category. In this section we are considering only functors of one variable (see also Part III, §3).

Definition. A connected sequence of covariant fu tors $T=\left\{T^{n}\right\}$ is a family of functors such that for every exact sequence

$$
\varnothing \rightarrow A^{\prime} \rightarrow A \rightarrow A^{\prime \prime} \rightarrow \varnothing,
$$


we have $T^{n}\left(A^{\prime \prime}\right) \rightarrow T^{n+1}\left(A^{\prime}\right)$ such that

$$
\cdots \rightarrow T^{n}(A) \rightarrow T^{n}\left(A^{\prime \prime}\right) \rightarrow T^{n+1}\left(A^{\prime}\right) \rightarrow T^{n+1}(A) \rightarrow \cdots
$$

is of order two, and for every commutative diagram

$$
\begin{aligned}
& \varnothing \rightarrow A_{1} \rightarrow A \rightarrow A_{2} \rightarrow \varnothing \\
& \varnothing \rightarrow B_{1} \rightarrow \grave{\downarrow} \rightarrow B_{2} \rightarrow \varnothing
\end{aligned}
$$

with exact rows,

$$
\begin{aligned}
T^{n}\left(A^{\prime \prime}\right) & \rightarrow T^{n+1}\left(A^{\prime}\right) \\
\downarrow & \downarrow \\
T^{n}\left(B^{\prime \prime}\right) & \rightarrow T^{n+1}\left(B^{\prime}\right)
\end{aligned}
$$

is commutative.

For contravariant functors, $A^{\prime}$ and $A^{\prime \prime}$ should be interchanged.

Let us consider the exact sequence

$$
\varnothing \rightarrow A^{0} \rightarrow A^{1} \rightarrow \cdots \rightarrow A^{p} \rightarrow \varnothing, \quad p>1 .
$$

Then we obtain the iterated connecting map $T^{n-p}\left(A^{p}\right) \rightarrow T^{n-1}\left(A^{0}\right)$ in the following way. We may consider the exact sequences

$$
\begin{aligned}
& \varnothing \rightarrow Z^{p-1} \rightarrow A^{p-1} \rightarrow A^{p} \rightarrow \varnothing, \\
& \varnothing \rightarrow Z^{p-2} \rightarrow A^{p-2} \rightarrow Z^{p-1} \rightarrow \varnothing, \\
& . . . . . \cdots \cdot \cdot \cdot \\
& \varnothing \rightarrow A^{0} \rightarrow A^{1} \rightarrow Z^{2} \rightarrow \varnothing .
\end{aligned}
$$

We have

$$
T^{n-p}\left(A^{p}\right) \rightarrow T^{n-(p-1)}\left(Z^{p-1}\right) \rightarrow \cdots \rightarrow T^{n-1}\left(A^{0}\right) .
$$

The iterated connecting map is defined as the composition of these maps.

Proposition 4.1. Consider the diagram (D) of Part I, 5.5. Assume that all rows and columns are exact. Then for $T=\left\{T^{*}\right\}$ a connected sequence of functors, we have

$$
\begin{aligned}
T^{n-1}\left(C_{2}\right) & \stackrel{\phi_{1}}{\rightarrow} T^{n}\left(A_{2}\right) \\
\phi_{2} \downarrow & \downarrow \psi_{1} \\
T^{n}\left(C_{1}\right) & \stackrel{\psi_{2}}{\rightarrow} T^{n+1}\left(A_{1}\right)
\end{aligned}
$$

with $\psi_{1} \phi_{1}+\psi_{2} \phi_{2}=0$.

Proof. We shall use the notation of Part I, 5.5. Before praceeding with the proof of 4.1, we must first consider some more exact sequences obtainable from (D) which are essentially dual to the ones obtained in the proof of Part I, 5.5. 


$$
\begin{aligned}
& \varnothing \rightarrow B_{1} \stackrel{\eta}{\rightarrow} B \stackrel{\zeta}{\rightarrow} B_{2} \rightarrow \varnothing \quad \varnothing \rightarrow A \stackrel{\kappa}{\rightarrow} B \stackrel{\rho}{\rightarrow} C \rightarrow \varnothing \\
& \varnothing \rightarrow A_{2} \stackrel{\kappa_{2}}{\rightarrow} B_{2} \stackrel{\rho_{2}}{\rightarrow} C_{2} \rightarrow \varnothing \quad \varnothing \rightarrow C_{1} \stackrel{\alpha}{\rightarrow} C \stackrel{\beta}{\rightarrow} C_{2} \rightarrow \varnothing \\
& \varnothing \rightarrow K \stackrel{\bar{\sigma}}{\rightarrow} B \stackrel{\rho_{2} \zeta}{\rightarrow} C_{2} \rightarrow \varnothing \quad \varnothing \rightarrow K \stackrel{\bar{\sigma}}{\rightarrow} B \stackrel{\rho_{2} \zeta}{\rightarrow} C_{2} \rightarrow \varnothing \\
& \varnothing \rightarrow B_{1} \stackrel{u_{1}}{\rightarrow} K \stackrel{v_{1}}{\rightarrow} A_{2} \rightarrow \varnothing \quad \varnothing \rightarrow A \stackrel{u_{2}}{\rightarrow} K \stackrel{v_{2}}{\rightarrow} C_{1} \rightarrow \varnothing \\
& \bar{\sigma} u_{1}=\eta \\
& \zeta \bar{\sigma}=\kappa_{2} v_{1} \\
& \bar{\sigma} u_{2}=x \\
& \rho \bar{\sigma}=\alpha \nu_{2} \\
& \varnothing \rightarrow A \stackrel{u_{2}}{\rightarrow} K \stackrel{v_{2}}{\rightarrow} C_{1} \rightarrow \varnothing \quad \varnothing \rightarrow B_{1} \stackrel{u_{1}}{\rightarrow} K \stackrel{v_{1}}{\rightarrow} A_{2} \rightarrow \varnothing \\
& \varnothing \rightarrow A_{1} \stackrel{\lambda}{\rightarrow} A \stackrel{\mu}{\rightarrow} A_{2} \rightarrow \varnothing \quad \varnothing \rightarrow A_{1} \stackrel{\kappa_{1}}{\rightarrow} B_{1} \stackrel{\rho_{1}}{\rightarrow} C_{1} \rightarrow \varnothing \\
& \varnothing \rightarrow A_{1} \stackrel{u_{2} \lambda}{\rightarrow} K \stackrel{\pi}{\rightarrow} F \rightarrow \varnothing \quad \varnothing \rightarrow A_{1} \stackrel{u_{2} \lambda}{\rightarrow} K \stackrel{\pi}{\rightarrow} F \rightarrow \varnothing \\
& \varnothing \rightarrow A_{2} \stackrel{u_{3}}{\rightarrow} F \stackrel{v_{3}}{\rightarrow} C_{1} \rightarrow \varnothing \quad \varnothing \rightarrow C_{1} \stackrel{u_{4}}{\rightarrow} F \stackrel{v_{4}}{\rightarrow} A_{2} \rightarrow \varnothing \\
& u_{3} \mu=\pi u_{2} \\
& u_{4} \rho_{1}=\pi u_{1} \\
& v_{3} \pi=\nu_{2} \\
& v_{4} \pi=v_{1}
\end{aligned}
$$

It can be easily verified that $v_{4} u_{3}=e_{A_{2}}, v_{3} u_{4}=e_{C_{1}}$.

Now consider $R$ of Part I, 5.5. We have $d=u_{4} m_{3}+u_{8} m_{4}: R \rightarrow F . d$ is an equivalence.

The following diagrams are commutative:

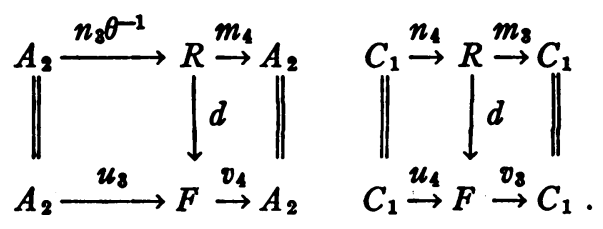

We now proceed with the proof of the theorem. First we show that $n_{8} \theta^{-1} m_{4}+n_{4} m_{3}=e_{R} ; u_{3} v_{4}+u_{4} v_{3}=e_{P}$.

Let $n_{3} \theta^{-1} m_{4}+n_{4} m_{3}+\chi=e_{R}$. Then $\chi n_{4}=0$, so $\chi=\chi^{\prime} m_{4}$. Thus $\left(\chi^{\prime}+n_{3} \theta^{-1}\right) m_{4}$ $+n_{4} m_{3}=e_{R}$ and $\chi^{\prime}+n_{3} \theta^{-1}=n_{3} \theta^{-1}$. Hence $\chi^{\prime}=0$ and $\chi=0$. Similarly $u_{3} v_{4}+u_{4} v_{3}$ $=e_{p}$.

The commutative diagrams

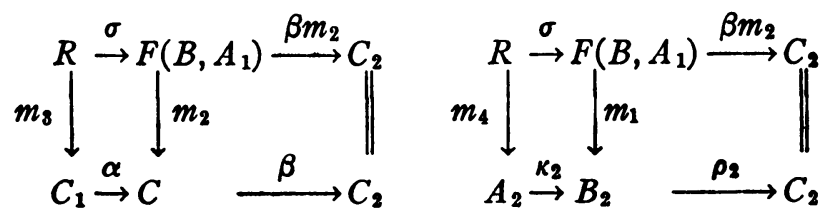




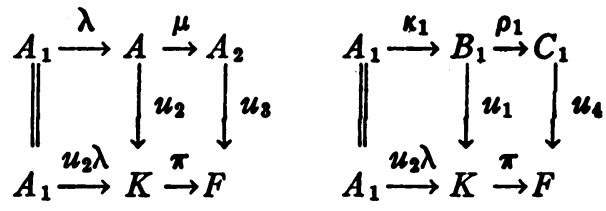

and the connectedness of $T$ will yield

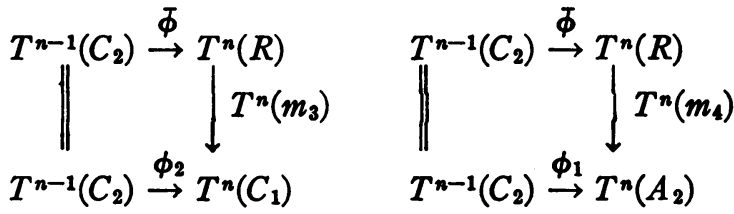

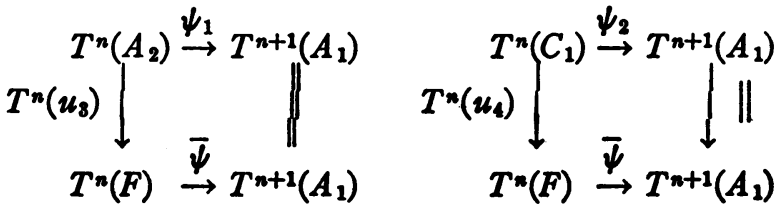

commutative.

$\psi_{1} \phi_{1}+\psi_{2} \phi_{2}=\Psi T^{n}(d) \Phi$. Hence we must merely show that $\Psi T^{n}(d) \phi=0$. Let us consider the diagram

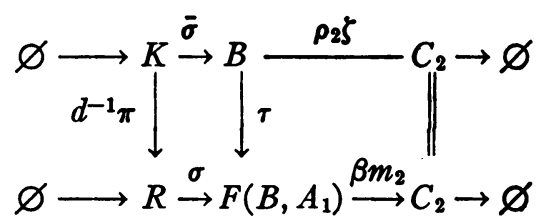

The right box is commutative. If we show that the left box is commutative, we shall obtain

$$
\begin{aligned}
& \prod^{n-1}\left(C_{2}\right) \stackrel{\bar{f}}{\rightarrow} T^{n}\left\{\begin{array}{l}
K) \\
T^{n}\left(d^{-1}\right) T^{n}(\pi)
\end{array}\right. \\
& T^{n-1}\left(C_{2}\right) \stackrel{\Phi}{\rightarrow} T^{n}(R)
\end{aligned}
$$

commutative. But then $\psi T^{n}(d) \Phi=\psi T^{n}(\pi) f^{n}=0$.

Therefore we shall show that $\sigma d^{-1} \pi=\tau \bar{\sigma}$. Since $\bar{\sigma} u_{2} \lambda=\eta \kappa_{1}$, we have a unique $\operatorname{map} q: F \rightarrow F\left(B, A_{1}\right)$ such that $q \bar{\pi}=\tau \bar{\sigma}$. Now $\beta m_{2} q \pi=\beta m_{2} \tau \bar{\sigma}=\beta \rho \bar{\sigma}=\beta \alpha v_{2}=0$, hence $\beta m_{2} q=0$ and $q=\sigma d^{\prime}, d^{\prime}: F \rightarrow R$.

$$
\sigma d^{\prime} u_{8} \mu=q \pi u_{2}=\tau \bar{\sigma} u_{2}=\tau \kappa=n_{2} \theta^{-1} \mu=\sigma n_{3} \theta^{-1} \mu .
$$

Hence $d^{\prime} u_{3}=n_{8} \theta^{-1}$. Also $d^{\prime} u_{4}=n_{4}$. 
Let $d d^{\prime}=e_{F}+\chi$. Then, $d d^{\prime} u_{3}=u_{3}+\chi u_{3}$ and $d d^{\prime} u_{3}=d n_{3} \theta^{-1}=u_{3}$. Thus, $\chi u_{3}=0$, and $\chi=\chi^{\prime} v_{3}$. We have, then, $d d^{\prime}=e_{F}+\chi^{\prime} v_{3}$. Now

$$
u_{4}=d n_{4}=d d^{\prime} u_{4}=u_{4}+\chi^{\prime} v_{3} u_{4}=u_{4}+\chi^{\prime} \text {. }
$$

Hence $\chi^{\prime}=0$ and $\chi=0$. Therefore $d d^{\prime}=e_{F}$ and $d^{\prime}=d^{-1}$.

Since $\sigma d^{-1} \pi=\sigma d^{\prime} \pi=q \pi=\tau \bar{\sigma}$, the proof is complete.

Let $(A, d)$ be an object with differentiation, and let $T$ be a covariant functor. Consider the commutative diagram

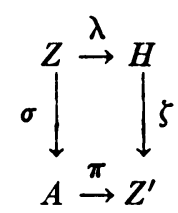

Now $Z, Z^{\prime}$, and $H$ can be considered objects with differentiation, the differentiation being zero. Under those conditions, all the maps in (2) are maps of objects with differentiation. Also, we see that $H(T(Z))=T(Z)$, $H(T(H))=T(H), H\left(T\left(Z^{\prime}\right)\right)=T\left(Z^{\prime}\right)$. Let us consider the induced commutative diagram

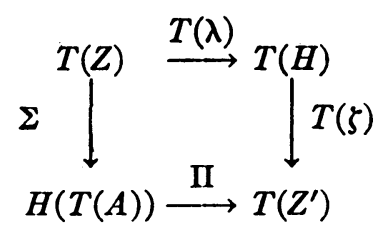

where $\Sigma=[T(\sigma)]_{*}, \Pi=[T(\pi)]_{*}$.

Proposition 4.2. Let $T$ be right exact (see Part III, 3). Then there exists a unique map $\alpha: T(H) \rightarrow H(T(A))$ such that $\alpha T(\lambda)=\Sigma$ and $\Pi \alpha=T(\zeta)$. $\alpha$ is natural relative to maps $A \rightarrow \bar{A}$ and is the identity on objects with zero differentiation. Furthermore, these last two properties characterize $\alpha$ completely.

Proof. Since $T$ is right exact,

$$
T\left(B^{\prime}\right) \stackrel{T(\omega)}{\longrightarrow} T(Z) \stackrel{T(\lambda)}{\longrightarrow} T(H) \rightarrow \varnothing
$$

is exact. We must show that $\Sigma T(\omega)=0$. It is easy to see that $\Sigma T(\omega)=[T(\kappa)]_{*}$. Also, since $T$ is right exact, $T(\theta \tau)$ is an epimorphism. Now we have the commutative diagrams
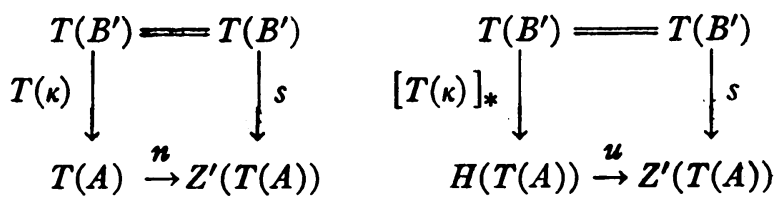
$u(T(\kappa))_{*}=n T(\kappa)$, thus $u(T(\kappa))_{*} T(\theta \tau)=n T(d)=0$, since $Z^{\prime}(T(A))=$ Coker $T(d)$. Therefore $[T(k)]_{*}=0=\Sigma T(\omega)$. Thus we have a map $\alpha: T(H) \rightarrow H(T(A))$ with $\alpha T(\lambda)=\Sigma$. To show that $\Pi \alpha=T(\zeta)$, we observe that $\Pi \alpha T(\lambda)=\Pi \Sigma$ $=T(\zeta) T(\lambda)$, and in view of the fact that $T(\lambda)$ is an epimorphism, we have $\Pi \alpha=T(\zeta)$.

Suppose we have $f:(A, d) \rightarrow(\bar{A}, \bar{d})$. We must show that

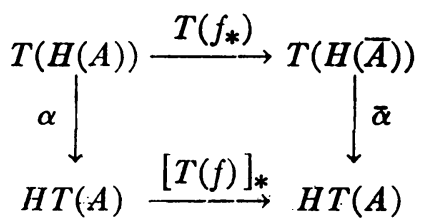

is commutative.

$$
\begin{aligned}
\bar{u}[T(f)]_{*} \alpha T(\lambda) & =\bar{u}[T(f)]_{*} \Sigma \\
\bar{u} \bar{\alpha} T\left(f_{*}\right) T(\lambda) & =\bar{u} \bar{\alpha} T(\bar{\lambda}) T(z)=\bar{u}[T(\bar{\sigma})]_{*} T(z) \\
& =\bar{n} T(\bar{\sigma}) T(z)=\bar{n} T(f) T(\sigma)=\bar{u}[T(f)]_{*}[T(\sigma)]_{*}=\bar{u}\left[\left.T(f)\right|_{*} \Sigma\right.
\end{aligned}
$$

Let $A$ have zero differentiation. Then we have

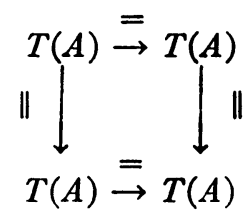

and $\alpha$ is obviously the identity.

To prove that these properties completely characterize $\alpha$, suppose $\alpha, \beta$ : $T(H(A)) \rightarrow H(T(A))$ both are natural and are the identity on objects with zero differentiation. Then $\alpha T(\lambda)=\Sigma=\beta T(\lambda)$ implies $\alpha=\beta$.

\section{Part III. Derived Functors}

1. Direct sums. Up to this point, we have carefully avoided using direct sums (although Part I, 5.5 and Part II, 4.1 certainly indicate their use). As yet, we have found no efficient way of defining infinite direct sums and products in an arbitrary exact category $\mathcal{A}$.

Definition. A family of maps

$$
A_{\alpha} \stackrel{l_{\alpha}}{\rightarrow} A \stackrel{p_{\alpha}}{\rightarrow} A_{\alpha},
$$

where $\alpha$ belongs to a finite set of indices, is a direct sum representation of $A$ if

$$
p_{\alpha} l_{\alpha}=e_{A_{\alpha}}, p_{\beta} l_{\alpha}=0 \text { for } \beta \not \alpha, \sum_{\alpha} l_{\alpha} p_{\alpha}=e_{A} \text {. }
$$

This definition does not guarantee the existence of a direct sum of given 
summands. For this purpose we introduce

Axiom $\mathrm{V}$ (Existence of direct sums). For any two objects $A_{1}, A_{2} \in \mathcal{A}$, there is an object $A \in \mathcal{A}$ and maps

$$
A_{\alpha} \stackrel{l_{\alpha}}{\rightarrow} A \stackrel{p_{\alpha}}{\rightarrow} A_{\alpha}
$$$$
\alpha=1,2,
$$

which yield a direct sum representation of $A$.

This axiom is obviously self-dual.

We easily deduce that the direct sum of a finite number of objects in $\mathcal{A}$ exists in $\mathcal{A}$, and any two such are equivalent.

Definition. We say the exact sequence $\varnothing \rightarrow A_{1} \rightarrow^{\alpha} A \rightarrow^{\beta} A_{2} \rightarrow \varnothing$ rightsplits if there is a map $\gamma: A_{2} \rightarrow A$ such that $\beta \gamma=e_{\Lambda_{2}}$. We say the sequence leftsplits if there is a $\delta: A \rightarrow A_{1}$ such that $\delta \alpha=e_{A_{1}}$.

Proposition 1.1. The sequence $\varnothing \rightarrow A_{1} \rightarrow^{\alpha} A \rightarrow^{\beta} A_{2} \rightarrow \varnothing$ right-splits if and only if it left-splits and in either case we can find maps such that

$$
\begin{aligned}
& A_{1} \stackrel{\alpha}{\rightarrow} A \stackrel{\delta}{\rightarrow} A_{1}, \\
& A_{2} \stackrel{\gamma}{\rightarrow} A \stackrel{\beta}{\rightarrow} A_{2}
\end{aligned}
$$

yield a direct sum representation of $A$.

Remark. Referring to (D) of Part I, 5.5, we have (as was indicated at the end of that proof)

$\operatorname{Ker}\left(\operatorname{Coker}(\kappa \lambda) \rightarrow C_{2}\right)$ is the direct sum of $C_{1}$ and $A_{2}$. So also is

$$
\text { Coker }\left(A_{1} \rightarrow \operatorname{Ker}(\beta \rho)\right) \text {. }
$$

Proposition 1.2. Consider the commutative diagram

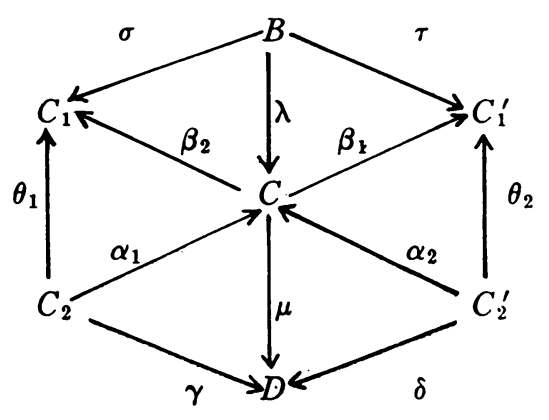

with diagonals exact, $\theta_{1}, \theta_{2}$ equivalences, and $\mu \lambda=0$. Then

and

$$
C_{2} \stackrel{\alpha_{1}}{\rightarrow} C \stackrel{\theta_{1}^{-1} \beta_{2}}{\longrightarrow} C_{2}, \quad C_{2}^{\prime} \stackrel{\alpha_{2}}{\rightarrow} C \stackrel{\theta_{2}^{-1} \beta_{1}}{\longrightarrow} C_{2}^{\prime}
$$




$$
C_{1} \stackrel{\alpha_{1} \theta_{1}^{-1}}{\longrightarrow} C \stackrel{\beta_{2}}{\rightarrow} C_{1}, \quad C_{2}^{\prime} \stackrel{\alpha_{2} \theta_{2}^{-1}}{\longrightarrow} C \stackrel{\beta_{1}}{\rightarrow} C_{1}^{\prime}
$$

yield direct sum representations of $C$. Furthermore

$$
\gamma \theta_{1}^{-1} \sigma+\delta \theta_{2}^{-1} \tau=0 .
$$

Let $\varnothing \rightarrow A_{1} \rightarrow^{\alpha_{1}} B_{1} \rightarrow^{\beta_{1}} C \rightarrow \varnothing, \varnothing \rightarrow A_{2} \rightarrow^{\alpha_{2}} B_{2} \rightarrow{ }^{\beta_{2}} C \rightarrow \varnothing$ be exact, and let

$$
B_{1} \stackrel{l_{1}}{\rightarrow} B_{1}+B_{2} \stackrel{p_{1}}{\rightarrow} B_{1} \quad B_{2} \stackrel{l_{2}}{\rightarrow} B_{1}+B_{2} \stackrel{p_{2}}{\rightarrow} B_{2}
$$

be a direct sum representation. We obtain the map $\beta_{1} p_{1}-\beta_{2} p_{2}: B_{1}+B_{2} \rightarrow C$ and the exact sequence

$$
\varnothing \rightarrow R \stackrel{\sigma}{\rightarrow} B_{1}+B_{2} \stackrel{\beta_{1} p_{1}-\beta_{2} p_{2}}{\longrightarrow} C \rightarrow \varnothing .
$$

Since $\left(\beta_{1} p_{1}-\beta_{2} p_{2}\right) l_{j} \alpha_{j}=0, j=1,2$, we have $\kappa_{1}: A_{1} \rightarrow R, \kappa_{2}: A_{2} \rightarrow R$ with $\sigma \kappa_{j}=l_{j} \alpha_{j,}$ $j=1,2$.

LEMMA 1.3. The sequences

$$
\begin{aligned}
& \varnothing \rightarrow A_{1} \stackrel{\kappa_{1}}{\rightarrow} R \stackrel{\stackrel{p_{2} \sigma}{\longrightarrow}}{\rightarrow} B_{2} \rightarrow \varnothing, \\
& \varnothing \rightarrow A_{2} \stackrel{\kappa_{2}}{\rightarrow} R \stackrel{p_{1} \sigma}{\longrightarrow} B_{1} \rightarrow \varnothing
\end{aligned}
$$

are exact.

\section{Projectives and injectives.}

Definition. An object $P \in \mathcal{A}$ is called projective if any diagram

$$
\underset{A \stackrel{g}{\rightarrow}}{\left.\right|^{\prime \prime} \rightarrow \varnothing}
$$

in which the row is exact may be imbedded in a commutative diagram

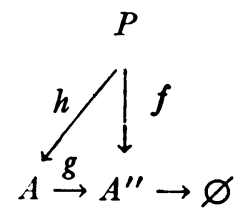

We define $Q \in \mathcal{A}$ to be injective by duality.

For further work, we need the following axiom and its dual:

Axıom VI (Existence of projectives). Given $A \in \mathcal{A}$, there is an epimorphism $P \rightarrow A$ with $P$ projective.

Proposition 2.1. A direct sum of objects is projective if and only if each summand is projective. 
Proposition 2.2. In order that $P$ be projective, it is necessary and sufficient that all exact sequences $\varnothing \rightarrow A^{\prime} \rightarrow A \rightarrow P \rightarrow \varnothing$ split.

Proposition 2.3. Let $\varnothing \rightarrow A^{\prime} \rightarrow^{\alpha} A \rightarrow^{\beta} A^{\prime \prime} \rightarrow \varnothing$ be exact. Consider the diagram

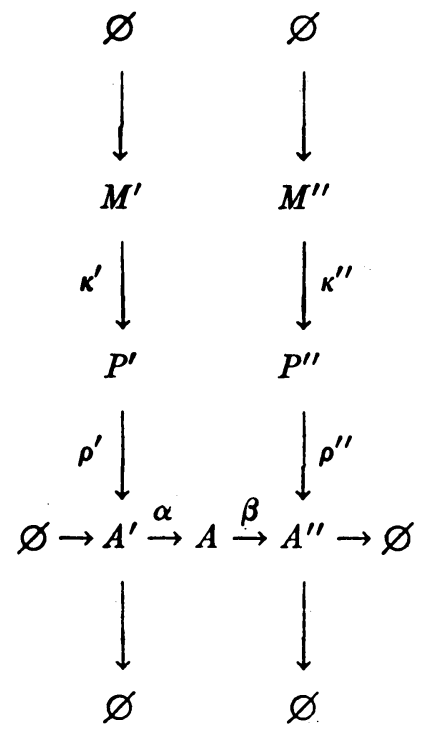

with exact columns, $P^{\prime}$ and $P^{\prime \prime}$ projective. Then this diagram can be imbeddec in the commutative diagram,

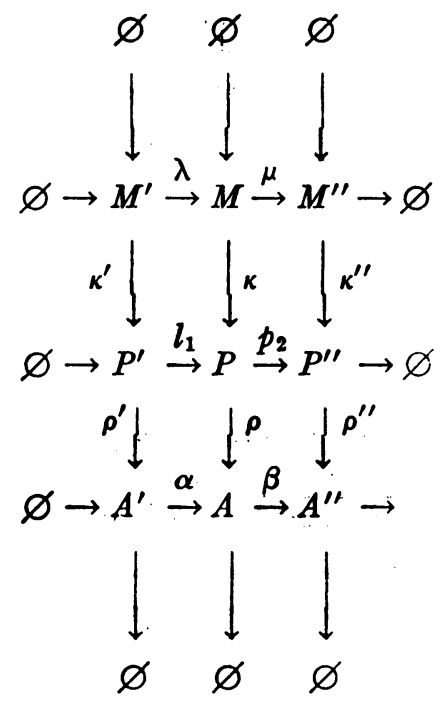


in which rows and columns are exact, and the middle row splits (therefore $P$ is projective).

Proof. We let $P=P^{\prime}+P^{\prime \prime}$. Then

$$
P^{\prime} \stackrel{l_{1}}{\rightarrow} P \stackrel{p_{1}}{\rightarrow} P^{\prime}, \quad P^{\prime \prime} \stackrel{l_{2}}{\rightarrow} P \stackrel{p_{2}}{\rightarrow} P^{\prime \prime}
$$

is a direct sum representation of $P$. Since $P^{\prime \prime}$ is projective, we have $\gamma: P^{\prime \prime} \rightarrow A$ with $\beta \gamma=\rho^{\prime \prime}$. Define $\rho=\alpha \rho^{\prime} p_{1}+\gamma p_{2}: P \rightarrow A$. Then $\rho l_{1}=\alpha \rho^{\prime}, \beta \rho=\rho^{\prime \prime} p_{2} . \rho$ is an epimorphism, and the remainder of the proof is automatic, if we choose $M=\operatorname{Ker} \rho$.

3. Functors of several variables. For $A \in \mathcal{A}, C \in \mathcal{C}$, we consider functors $T(A, C)$ covariant in $A$, contravariant in $C$, with values in $D$, in the sense of [2]. Throughout we assume the functors additive, in that

$$
\begin{aligned}
& T\left(\alpha+\beta, e_{C}\right)=T\left(\alpha, e_{C}\right)+T\left(\beta, e_{C}\right), \\
& T\left(e_{A}, \gamma+\delta\right)=T\left(e_{A}, \gamma\right)+T\left(e_{A}, \delta\right) .
\end{aligned}
$$

Definition. Let $\mathcal{A}, \mathcal{C}, \mathcal{D}$ be three exact categories.

Let

$$
\begin{aligned}
& \varnothing \rightarrow A_{1} \rightarrow A \rightarrow A_{2} \rightarrow \varnothing, \\
& \varnothing \rightarrow C_{1} \rightarrow C \rightarrow C_{2} \rightarrow \varnothing
\end{aligned}
$$

be exact.

Definition. A functor $T$ is exact if for all exact sequences (1) and (2)

$$
\begin{aligned}
& \varnothing \rightarrow T\left(A_{1}, C\right) \rightarrow T(A, C) \rightarrow T\left(A_{2}, C\right) \rightarrow \varnothing, \\
& \varnothing \rightarrow T\left(A, C_{2}\right) \rightarrow T(A, C) \rightarrow T\left(A, C_{1}\right) \rightarrow \varnothing
\end{aligned}
$$

are exact.

$T$ is right exact if for all sequences (1) and (2)

$$
\begin{aligned}
& T\left(A_{1}, C\right) \rightarrow(T, C) \rightarrow T\left(A_{2}, C\right) \rightarrow \varnothing, \\
& T\left(A, C_{2}\right) \rightarrow T(A, C) \rightarrow T\left(A, C_{1}\right) \rightarrow \varnothing
\end{aligned}
$$

are exact.

Analogous definition for left exact.

$T$ is half exact if for all sequences (1) and (2)

$$
\begin{aligned}
& T\left(A_{1}, C\right) \rightarrow T(A, C) \rightarrow T\left(A_{2}, C\right), \\
& T\left(A, C_{2}\right) \rightarrow T(A, C) \rightarrow T\left(A, C_{1}\right)
\end{aligned}
$$

are exact.

The usual discussion about composition of functors and preservation of exactness under composition [1, II. 5] applies. However, we single out two cases for special study. 
Let $D$ denote generically the functor which takes an exact category $\mathcal{A}$ into its dual $\mathcal{A}^{*}$. $T$ is a functor.

We define $T_{*}$ by

$$
T_{*}\left(A, C^{*}\right)=T\left(A, D^{-1}\left(C^{*}\right)\right)=T(A, C) .
$$

Since $D,\left(D^{-1}\right)$, is an exact, contravariant functor, $T$ and $T_{*}$ differ in variance on $C$ but preserve the same type of exactness. We see that $\left(T_{*}\right)_{*}=T$ (more precisely, $\left(T_{*}\right)_{*}$ is naturally equivalent to $\left.T\right)$.

We define $T^{*}$ by

$$
T^{*}\left(A^{*}, C\right)=D\left[T\left(D^{-1}\left(A^{*}\right), C\right)\right] .
$$

$T^{*}$ and $T$ differ in their variance on $C$ and also preserve opposite types of exactness (i.e., when $T$ is left exact, $T^{*}$ is right exact; $T$ exact, $T^{*}$ exact; etc.).

\section{Satellites of functors.}

Definition. Let

$$
\varnothing \rightarrow M \rightarrow P \rightarrow A \rightarrow \varnothing
$$

be exact, with $P$ projective. Let $T$ be a covariant functor of one variable. The left satellite of $T$, denoted by $S_{1} T(A)$, is defined as $\operatorname{Ker}(T(M) \rightarrow T(P))$.

It is shown in $[1$, III $]$ that $S_{1} T$ is independent (up to a transitive family of equivalences) of the choice of the sequence $\varnothing \rightarrow M \rightarrow P \rightarrow A \rightarrow \varnothing$.

Now let $g: A \rightarrow A^{\prime}$. We then have

$$
\begin{aligned}
\varnothing \rightarrow M & \rightarrow P \\
\downarrow & \rightarrow A \rightarrow \varnothing \\
\varnothing \rightarrow M^{\prime} \rightarrow & \downarrow P^{\prime} \rightarrow A^{\prime} \rightarrow \varnothing
\end{aligned}
$$

with $P$ and $P^{\prime}$ projective. But then we obtain

$$
\begin{array}{ccc}
\varnothing \rightarrow S_{1} T(A) & \rightarrow T(M) & \rightarrow T(P) \\
\downarrow & \downarrow & \downarrow \\
\varnothing \rightarrow S_{1} T\left(A^{\prime}\right) & \rightarrow T\left(M^{\prime}\right) & \rightarrow T\left(P^{\prime}\right)
\end{array}
$$

and we call the left-hand map $S_{1} T(\mathrm{~g})$.

With these definitions, $S_{1} T$ becomes a covariant functor.

Definitions. Let $T$ be a covariant functor. We define the right satellite of $T, S^{1} T$, by

$$
S^{1} T=\left(S_{1} T^{*}\right)^{*}
$$

i.e.,

$$
S^{1} T(A)=D^{-1}\left(S_{1} T^{*}(D(A))\right) .
$$

Let $T$ be a contravariant functor. Then $T_{*}$ is covariant. We define 


$$
\begin{aligned}
& S_{1} T(A)=S_{1} T_{*}\left(A^{*}\right), \\
& S^{1} T(A)=S^{1} T_{*}\left(A^{*}\right) .
\end{aligned}
$$

Due to these relationships, it is sufficient to consider only covariant functors.

DEFINITION.

$$
\begin{array}{llrl}
S_{n} T & =S_{1}\left(S_{n-1} T\right), & & n>1, \\
S^{n} T & =S^{1}\left(S^{n-1} T\right), & & n>1 .
\end{array}
$$

Let us consider the commutative diagram

$$
\begin{aligned}
& \varnothing \rightarrow A_{1} \stackrel{\alpha_{1}}{\rightarrow} A \stackrel{\alpha_{2}}{\rightarrow} A_{2} \rightarrow \varnothing \\
& \rho^{\prime} \downarrow \downarrow \rho \downarrow \rho_{2} \\
& \varnothing \rightarrow B_{1} \stackrel{\beta_{1}}{\rightarrow} B \stackrel{\beta_{2}}{\rightarrow} B_{2} \rightarrow \varnothing
\end{aligned}
$$

with exact rows.

We have

$$
\begin{aligned}
& \varnothing \rightarrow M_{2} \stackrel{\iota}{\rightarrow} P_{2} \stackrel{p}{\rightarrow} A_{2} \rightarrow \varnothing \\
& \mu \downarrow \downarrow \lambda \mid \\
& \varnothing \rightarrow A_{1} \stackrel{\alpha_{1}}{\rightarrow} A \stackrel{\alpha_{2}}{\rightarrow} A_{2} \rightarrow \varnothing
\end{aligned}
$$

and therefore a map $\Im_{1}\left(e_{A_{2}}\right): S_{1} T\left(A_{2}\right) \rightarrow \operatorname{Ker}\left(T\left(\alpha_{1}\right)\right)$. We define

$$
\theta_{1}=\sigma_{1} \Im_{1}\left(e_{A_{2}}\right): S_{1} T\left(A_{2}\right) \rightarrow T\left(A_{1}\right),
$$

where $\sigma_{1}: \operatorname{Ker}\left(T\left(\alpha_{1}\right)\right) \rightarrow T\left(A_{1}\right)$ is the "inclusion."

Dually, we obtain $\theta^{1}: T\left(A_{2}\right) \rightarrow S^{1} T\left(A_{1}\right)$. Thus we have

$$
\begin{aligned}
& \theta_{n}: S_{n} T\left(A_{2}\right) \rightarrow S_{n-1} T\left(A_{1}\right), \\
& \theta^{n}: S^{n-1} T\left(A_{2}\right) \rightarrow S^{n} T\left(A_{1}\right)
\end{aligned}
$$

for $n>0$ (we let $S_{0} T=S^{0} T=T$ ).

Consequently, we obtain the sequence

$$
\begin{aligned}
\cdots & \rightarrow S_{n} T\left(A_{1}\right) \rightarrow S_{n} T(A) \rightarrow S_{n} T\left(A_{2}\right) \rightarrow S_{n-1} T\left(A_{1}\right) \rightarrow \cdots \\
& \rightarrow S_{1} T\left(A_{2}\right) \rightarrow T\left(A_{1}\right) \rightarrow T(A) \rightarrow T\left(A_{2}\right) \\
& \rightarrow S^{1} T\left(A_{1}\right) \rightarrow \cdots \rightarrow S^{n-1} T\left(A_{2}\right) \rightarrow S^{n} T\left(A_{1}\right) \rightarrow \cdots
\end{aligned}
$$

Proposition 4.1. The sequence (4) is of order two.

Proposition 4.2. The diagram (2) induces a commutative diagram 


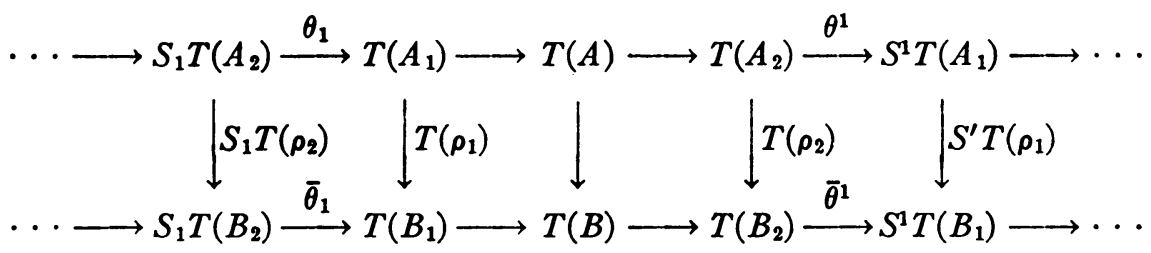

TheOREM 4.3. Let $T$ be a half-exact functor. Then the sequence (4) is exact.

\section{Axiomatic description of satellites.}

Definition. Let $T=\left\{T^{n}\right\}, U=\left\{U^{n}\right\}$ be connected sequences of functors (Part II, §4). A map $\Phi: T \rightarrow U$ is a family $\left\{\phi^{n}\right\}$ of natural transformations [2] $\phi^{n}: T^{n} \rightarrow U^{n}$ such that

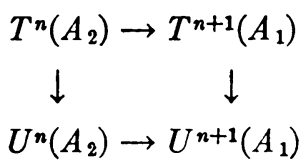

is commutative for every exact sequence $\varnothing \rightarrow A_{1} \rightarrow A \rightarrow A_{2} \rightarrow \varnothing$.

If $T$ is a functor, by $S T$ we mean the connected sequence of functors $S^{n} T$ where $S^{n} T=S^{n} T$ for $n \geqq 0, S^{n} T=S_{-n} T$ for $n<0$.

The axiomatic description of $S T$ can be found in [1]. However, we shall prove here a more general theorem which is applicable as well in the theory of sheaves.

Definition. Let $\mathcal{A}$ be an exact category (Axioms I-IV), let $\mathcal{Q}=\{Q\}$ be a subclass of the class of objects of $A$, and let $\phi:\{Q\} \rightarrow\{A\}$ be a (not necessarily single-valued) function from 2 to the objects of $\mathcal{A}$. We denote the pair $(2, \phi)$ by $2_{\phi}$. We say that $2_{\phi}$ is $\phi$-injective if every diagram

$$
\begin{gathered}
\varnothing \rightarrow \phi\left(Q_{1}\right) \rightarrow Q_{1} \\
\downarrow \\
\varnothing \rightarrow \phi\left(Q_{2}\right) \rightarrow Q_{2}
\end{gathered}
$$

with exact rows can be imbedded in a commutative diagram

$$
\begin{array}{cc}
\varnothing \rightarrow \phi\left(Q_{1}\right) & \rightarrow Q_{1} \\
\downarrow & \downarrow \\
\varnothing \rightarrow \phi\left(Q_{2}\right) & \rightarrow Q_{2} .
\end{array}
$$

We say a $\phi$-injective class is $\phi$-complete if $\phi$ is onto and for each $A \in \phi(Q)$, there is a monomorphism $A \rightarrow Q$.

TheOREM 5.1. Let $2_{\phi}$ be a $\phi$-complete $\phi$-injective class in $\mathcal{A}$. Let $T=\left\{T^{n}\right\}$ be a positive (i.e., $T^{n}=0$ for $\left.n<0\right)$ connected sequence of functors such that for every exact sequence 


$$
\varnothing \rightarrow A \rightarrow Q \rightarrow N \rightarrow \varnothing
$$

$Q \in 2, A \in \phi(Q)$

the sequence

$$
T^{n}(Q) \rightarrow T^{n}(N) \rightarrow T^{n+1}(A) \rightarrow \varnothing
$$$$
n \geqq 0
$$

is exact. Suppose, also, that $U=\left\{U^{n}\right\}$ is a positive connected sequence of functors and $\phi^{0}: T^{0} \rightarrow U^{0}$ is a natural transformation. Then there exists a unigue extension $\Phi: T \rightarrow U$ of $\phi^{0}$.

Proof. We proceed by induction on $n$. Suppose $\phi^{n}: T^{n} \rightarrow U^{n}$ has been defined. We consider an exact sequence of type (1) and obtain

$$
\begin{aligned}
T^{n}(Q) & \rightarrow T^{n}(N) \stackrel{\partial}{\rightarrow} T^{n+1}(A) \rightarrow \varnothing \\
\phi^{n}(Q){ }_{U^{n}(Q)} & \downarrow^{\rightarrow} U^{n}(N) \stackrel{\bar{\partial}}{\longrightarrow} U^{n+1}(A)
\end{aligned}
$$

commutative. Since the top row is exact, and the bottom row is of order two, we obtain a unique map $\phi^{n+1}(A): T^{n+1}(A) \rightarrow U^{n+1}(A)$ such that

$$
\phi^{n+1}(A) \partial=\bar{\partial} \phi^{n}(N) \text {. }
$$

This map appears to depend upon the sequence (1) which was originally chosen. Without changing notation, we shall consider $\phi^{n+1}(A)$ as a function of (1) as well as of $A$.

Let $f: A \rightarrow B$. Then

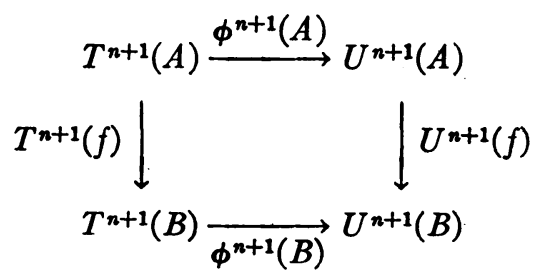

is commutative, i.e., $\phi^{n+1}$ is natural.

We choose any sequences

$$
\begin{aligned}
& \varnothing \rightarrow A \stackrel{\alpha}{\rightarrow} Q \stackrel{\beta}{\rightarrow} N \rightarrow \varnothing \\
& \downarrow \\
& \downarrow \\
& \varnothing \rightarrow B \stackrel{\bar{\alpha}}{\rightarrow} \stackrel{\bar{\beta}}{\rightarrow} \bar{N} \rightarrow \varnothing .
\end{aligned}
$$

Then we have $f^{\prime}: Q \rightarrow \bar{Q}$ with $f^{\prime} \alpha=\bar{\alpha} f$, and a unique $f^{\prime \prime}: N \rightarrow \bar{N}$ with $\beta f^{\prime}=f^{\prime \prime} \beta$. We therefore have the commutative diagrams 


$$
\begin{aligned}
& T^{n}(N) \stackrel{\partial}{\rightarrow} T^{n+1}(A) \\
& T^{n}\left(f^{\prime \prime}\right) \downarrow \quad T^{n+1}(f) \\
& T^{n}(\bar{N}) \stackrel{\partial}{\rightarrow} T^{n+1}(B) \\
& \phi^{n}(\bar{N}) \downarrow \quad \phi^{n+1}(B) \quad \phi^{n}(N) \downarrow \quad \downarrow \phi^{n}(\bar{N}) \\
& U^{n}(\bar{N}) \stackrel{\bar{\partial}}{\rightarrow} U^{n+1}(B) \quad U^{n}(N) \stackrel{U^{n}\left(f^{\prime \prime}\right)}{\longrightarrow} U^{n}(\bar{N}) \\
& U^{n}\left(f^{\prime \prime}\right) \uparrow \quad \uparrow U^{n+1}(f) \\
& U^{n}(N) \stackrel{\bar{\partial}}{\rightarrow} U^{n+1}(A)
\end{aligned}
$$

the second diagram being commutative by our induction hypothesis. Thus we have

$$
\begin{aligned}
& \phi^{n+1}(B) T^{n+1}(f) \partial=\phi^{n+1}(B) \partial T^{n}\left(f^{\prime \prime}\right)=\bar{\partial} \phi^{n}(\bar{N}) T^{n}\left(f^{\prime \prime}\right), \\
& U^{n+1}(f) \phi^{n+1}(A) \partial=U^{n+1}(f) \bar{\partial} \phi^{n}(N)=\bar{\partial} U^{n}\left(f^{\prime \prime}\right) \phi^{n}(N) .
\end{aligned}
$$

The right-hand sides being equal, and $\partial$ being an epimorphism, the desired result follows.

By choosing $A=B$, we see that $\phi^{n+1}$ is independent of the choice of the sequence (1).

If $\varnothing \rightarrow A^{\prime} \rightarrow^{\alpha} A \rightarrow^{\beta} A^{\prime \prime} \rightarrow \varnothing$ is exact, then

$$
\begin{aligned}
T^{n}\left(A^{\prime \prime}\right) & \stackrel{\partial^{\prime}}{\rightarrow} T^{n+1}\left(A^{\prime}\right) \\
\phi^{n}\left(A^{\prime \prime}\right) & \\
U^{n}\left(A^{\prime \prime}\right) & \stackrel{\bar{\partial}^{\prime}}{\rightarrow} U^{n+1}\left(A^{\prime}\right)
\end{aligned}
$$

is commutative.

Let $\varnothing \rightarrow A \rightarrow^{r} Q \rightarrow^{\delta} N \rightarrow \varnothing$ be exact. Then we have $\varnothing \rightarrow A^{\prime} \rightarrow^{\alpha \gamma} Q \rightarrow^{r} N^{\prime} \rightarrow \varnothing$ exact and we obtain the commutative diagram

$$
\begin{aligned}
& \varnothing \rightarrow A^{\prime} \stackrel{\gamma}{\rightarrow} A \stackrel{\beta}{\rightarrow} A^{\prime \prime} \rightarrow \varnothing
\end{aligned}
$$

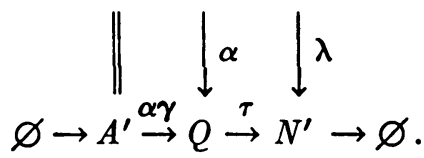


Consider the commutative diagrams

$$
\begin{aligned}
& T^{n}\left(A^{\prime \prime}\right) \stackrel{\partial^{\prime}}{\rightarrow} T^{n+1}\left(A^{\prime}\right) \\
& \left.T *(\lambda)\right|_{\downarrow} \quad \downarrow \| \\
& T^{n}\left(N^{\prime}\right) \stackrel{\partial^{\prime \prime}}{\rightarrow} T^{n+1}\left(A^{\prime}\right)
\end{aligned}
$$

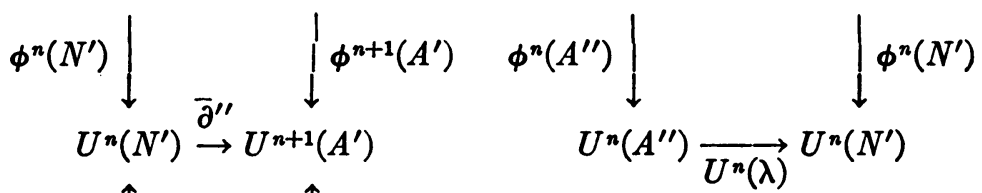

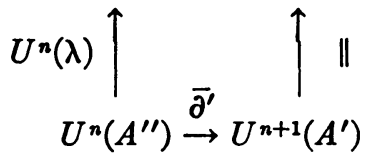

Then

$\bar{\partial}^{\prime} \phi^{n}\left(A^{\prime \prime}\right)=\bar{\partial}^{\prime \prime} U^{n}(\lambda) \phi^{n}\left(A^{\prime \prime}\right)=\bar{\partial}^{\prime \prime} \phi^{n}\left(N^{\prime}\right) T^{n}(\lambda)=\phi^{n+1}\left(A^{\prime}\right) \partial^{\prime \prime} T^{n}(\lambda)=\phi^{n+1}\left(A^{\prime}\right) \partial^{\prime}$.

\section{Derived functors.}

Definition. A doubly (multiply) graded or double (multiple) complex is a sequence $\left\{A^{p, q}\right\}$ of objects together with two sequences $\left\{d_{1}^{p, q}\right\},\left\{d_{2}^{p, q}\right\}$ of maps $d_{1}^{p, q}: A^{p, q} \rightarrow A^{p+1, q}, d_{2}^{p, q}: A^{p, q} \rightarrow A^{p, q+1}$ such that $d_{1}^{p+1, q} d_{1}^{p, q}=0=d_{2}^{p, q+1} d_{2}^{p, q}$, and $d_{1}^{p, q+1} d_{2}^{p, q}=d_{2}^{p+1, q} d_{1}^{p, q}$. Furthermore, for each $n$, all but a finite number of $A^{p, q}$ for which $p+q=n$ are different from zero.

Because of this last condition, we can define the associated singly graded complex in the usual way. The standard definitions of maps and homotopies can easily be made and the consequences in the associated complex deduced.

Definitions. Let $A \in \mathcal{A}$. By a left complex over $A$, we mean a complex

$$
\cdots \rightarrow A_{n} \rightarrow A_{n-1} \rightarrow \cdots \rightarrow A_{0} \stackrel{\epsilon}{\rightarrow} A \rightarrow \varnothing \rightarrow \varnothing \rightarrow \cdots .
$$

By an acyclic left complex over $A$, we mean a complex (1) such that the sequence (1) is exact.

By a projective left complex over $A$, we mean a complex (1) where each $A_{q}$ is projective.

By a projective resolution of $A$, we mean a projective, acyclic left complex over $A$.

Replacing "left" by "right," and "projective" by "injective," we obtain dual definitions.

The existence of projective resolutions of any object $A \in \mathcal{A}$ is ensured by Axiom VI.

Definition. Let $X, X^{\prime}$ be left complexes over $A$ and $C$ respectively and let $f: A \rightarrow C$. A map $F: X \rightarrow X^{\prime}$ is a map over $f$ if 


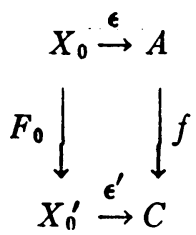

is commutative.

Proposition 6.1. Let $X$ be a projective left complex over $A$, and $X^{\prime}$ an acyclic left complex over $C$. Let $f: A \rightarrow C$. Then we can find a map $F: X \rightarrow X^{\prime}$ over $f$. Furthermore, any two such maps $F_{1}, F_{2}: X \rightarrow X^{\prime}$ over $f$ are chain homotopic.

Let $T$ be a covariant functor of two variables. Let $A \in \mathcal{A}, C \in \mathcal{C}, X, X^{\prime}$ projective resolutions of $A, C$ respectively. Then we get a double complex $T\left(X, X^{\prime}\right)$ defined by

$$
\begin{aligned}
T_{p, q}\left(X, X^{\prime}\right) & =T\left(X_{p}, X_{q,}^{\prime}\right), \\
d_{p, q}^{1} & =T\left(d_{p}, e_{X^{\prime} q}\right), \\
d_{p, q}^{2} & =T\left(e_{X_{p}}, d_{q}\right) .
\end{aligned}
$$

Definition. The $n$th left-derived functor, $L_{n} T(A, C)$ is $H_{n}\left(T\left(X, X^{\prime}\right)\right)$, where $H_{n}\left(T\left(X, X^{\prime}\right)\right)$ is the $n$th homology of the singly graded complex associated with the double complex $T\left(X, X^{\prime}\right)$.

Proposition 6.2. $L_{n} T(A, C)$ is independent (up to a transitive family of equivalences) of the choice of resolutions $X, X^{\prime}$.

Let $\alpha: A \rightarrow A^{\prime}, \gamma: C \rightarrow C^{\prime}$. Then in the natural way, we have a map

$$
L_{n} T(\alpha, \gamma): L_{n} T(A, C) \rightarrow L_{n} T\left(A^{\prime}, C^{\prime}\right) .
$$

Therefore, $L_{n} T$ is a functor of two variables.

Theorem 6.3. Let $\varnothing \rightarrow A_{1} \rightarrow A \rightarrow A_{2} \rightarrow \varnothing, \varnothing \rightarrow C_{1} \rightarrow C \rightarrow C_{2} \rightarrow \varnothing$ be exact. Then we have sequences

$$
\begin{aligned}
\cdots & \rightarrow L_{n} T\left(A_{1}, C\right) \rightarrow L_{n} T(A, C) \rightarrow L_{n} T\left(A_{2}, C\right) \rightarrow L_{n-1} T\left(A_{1}, C\right) \rightarrow \cdots \\
& \rightarrow L_{0} T\left(A_{1}, C\right) \rightarrow L_{0} T(A, C) \rightarrow L_{0} T\left(A_{2}, C\right) \rightarrow \varnothing \\
& \rightarrow L_{n} T\left(A, C_{1}\right) \rightarrow L_{n} T(A, C) \rightarrow L_{n} T\left(A, C_{2}\right) \rightarrow L_{n-1} T\left(A, C_{1}\right) \rightarrow \cdots \\
& \rightarrow L_{0} T\left(A, C_{1}\right) \rightarrow L_{0} T(A, C) \rightarrow L_{0} T\left(A, C_{2}\right) \rightarrow \varnothing
\end{aligned}
$$

which are exact [1].

Definition. Let $T$ be a covariant functor of two variables. Then $T^{*}\left(A^{*}, C^{*}\right)=[T(A, C)]^{*}$ is also covariant. Define

$$
R^{n} T(A, C)=\left[L_{n} T^{*}\left(A^{*}, C^{*}\right)\right]^{*} \text {. }
$$

$R^{n} T$ is called the $n$th right derived functor of $T$. Its formal properties can be 
deduced from the formal properties of $L_{n} T$.

Analyzing this definition, we see that $X^{*}, X^{\prime *}$ are projective resolutions of $A^{*}, C^{*}$ if and only if $X$ and $X^{\prime}$ are injective resolutions of $A$ and $C$. Thus

$$
\begin{aligned}
R^{n} T(A, C) & =\left[L_{n} T^{*}\left(A^{*}, C^{*}\right)\right]^{*}=\left[H_{n}\left(T^{*}\left(X^{*}, X^{\prime *}\right)\right)\right]^{*} \\
& =\left[H_{n}\left(\left(T\left(X, X^{\prime}\right)\right)^{*}\right]^{*}=H^{n}\left(T\left(X, X^{\prime}\right)\right),\right.
\end{aligned}
$$

which is precisely the definition of $R^{n} T(A, C)$ given in [1].

Definition. Let $T$ be a functor, covariant in $A$, contravariant in $C$. Then $T_{*}\left(A, C^{*}\right)=T(A, C)$ is covariant in $A$ and $C^{*}$. Define

$$
\begin{aligned}
L_{n} T(A, C) & =L_{n} T_{*}\left(A, C^{*}\right), \\
R^{n} T(A, C) & =R^{n} T_{*}\left(A, C^{*}\right) .
\end{aligned}
$$

An argument similar to the preceding one shows that these definitions agree with those given in [1].

The formal properties of the derived functors, their comparison with satellites, the notion of balanced functor as treated in [1] can be translated into the language of exact categories. Also, the maps $\alpha$ and $\alpha^{\prime}$ of Part II, $\$ 4$ can be defined abstractly for functors of several variables.

\section{Part IV. Applications}

1. The functors $E x t^{n}$. For each exact category $\mathcal{A}$, the functor $H(A, C)$ may be regarded as a functor contravariant in $A$, covariant in $C$, and with values in the exact category $\mathcal{X}$ of abelian groups. This functor is left exact; for a fixed $A_{0}, H\left(A_{0}, C\right)$ is an exact functor of $C$ if and only if $A_{0}$ is projective; for a fixed $C_{o} \in \mathcal{C}, H(A, C o)$ is exact in $A$ if and only if $C o$ is injective. Thus $H(A, C)$ is right balanced [1].

If $\mathcal{A}$ satisfies Axioms $\mathrm{V}$ and VI, then $\operatorname{Ext}^{n}(A, C)$ may be defined as the right derived functor with respect to the variable $A$ (i.e., using a projective resolution of $A$ ). If $A$ satisfies Axioms $\mathrm{V}$ and $\mathrm{VI}^{*}$, then injective resolutions of $C$ may be used to define $E x t^{n}(A, C)$. If $\mathcal{A}$ satisfies Axioms V, VI, and VI*, either or both may be used.

We can define the global dimension of an exact category as the highest integer $n$ for which $\operatorname{Ext}^{n}(A, C) \neq 0$. A category has global dimension zero if and only if $H(A, C)$ is exact, i.e., if all elements of $\mathcal{A}$ are projective (or injective). This takes the place of semi-simple rings in [1].

2. Axiomatic homology. The axiomatic homology and cohomology theories of Eilenberg-Steenrod [3] may be defined using an arbitrary exact category $\mathcal{A}$ as the range of values of the theory. Thus, replacing $\mathcal{A}$ by $\mathcal{A}^{*}$ replaces a homology theory by a cohomology theory and vice versa. This duality principle simplifies the exposition of the theory. Furthermore, the uniqueness proof [3, Chap. IV] remains valid for such generalized homology and cohomology theories. 
3. Pontrjagin duality. The Pontrjagin duality for discrete and compact abelian groups readily shows that the category $\mathcal{C}$ of compact abelian groups is the dual of the category $\mathscr{H}$ of discrete abelian groups. Thus we conclude that $\mathcal{C}$ satisfies Axioms V, VI, and VI*. In fact, in $\mathcal{C}$, the injectives are the toroids (since the only discrete abelian projectives are the free groups); and the projectives in $\mathcal{C}$ are those compact groups whose character groups are divisible.

\section{BIBLIOGRAPHY}

1. H. Cartan and S. Eilenberg, Homological algebra, Princeton University Press, 1955.

2. S. Eilenberg and S. MacLane, General theory of natural equivalences, Trans. Amer. Math. Soc. vol. 58 (1945) pp. 231-294.

3. S. Eilenberg and N. E. Steenrod, Foundations of algebraic topology, Princeton University Press, 1952.

4. S. MacLane, Duality for groups, Bull. Amer. Math. Soc. vol. 56 (1950) pp. 485-516.

Princeton University,

Princeton, N. J. 